\title{
SPH simulations of clumps formation by dissipative collision of molecular clouds
}

\section{Non magnetic case}

\author{
E.P. Marinho ${ }^{1}$ and J.R.D. Lépine ${ }^{2}$ \\ 1 Instituto de Geociências e Ciências Exatas, Departamento de Estatística, Matemática Aplicada e Computacional, UNESP, \\ Rua 10, 2527, 13500-230, Rio Claro SP, Brazil \\ 2 Instituto Astronômico e Geofiisico, Departamento de Astronomia, USP, Av. Miguel Stefano, 4200, 04301-904 São Paulo SP, \\ Brazil
}

Received September 7; accepted December 17, 1999

\begin{abstract}
Computer experiments of interstellar cloud collisions were performed with a new smoothed-particlehydrodynamics (SPH) code. The SPH quantities were calculated by using spatially adaptive smoothing lengths and the SPH fluid equations of motion were solved by means of a hierarchical multiple time-scale leapfrog. Such a combination of methods allows the code to deal with a large range of hydrodynamic quantities. A careful treatment of gas cooling by $\mathrm{H}, \mathrm{H}_{2}, \mathrm{CO}$ and $\mathrm{HII}$, as well as a heating mechanism by cosmic rays and by $\mathrm{H}_{2}$ production on grains surface, were also included in the code. The gas model reproduces approximately the typical environment of dark molecular clouds. The experiments were performed by impinging two dynamically identical spherical clouds onto each other with a relative velocity of $10 \mathrm{~km} \mathrm{~s}^{-1}$ but with a different impact parameter for each case. Each object has an initial density profile obeying an $r^{-1}$-law with a cutoff radius of $10 \mathrm{pc}$ and with an initial temperature of $20 \mathrm{~K}$. As a main result, cloud-cloud collision triggers fragmentation but in expense of a large amount of energy dissipated, which occurred in the head-on case only. Offcenter collision did not allow remnants to fragment along the considered time $(\sim 6 \mathrm{Myr})$. However, it dissipated a considerable amount of orbital energy. Structures as small as $0.1 \mathrm{pc}$, with densities of $\sim 10^{4} \mathrm{~cm}^{-3}$, were observed in the more energetic collision.
\end{abstract}

Key words: hydrodynamics - shock waves methods: numerical — ISM: clouds - ISM: kinematics and dynamics - ISM: molecules

Send offprint requests to: E.P. Marinho, e-mail: emarinho@rc.unesp.br

\section{Introduction}

Smoothed particle hydrodynamics was first introduced by Lucy (1977) and by Gingold \& Monaghan (1977), and nowadays it is the most convenient approach to simulate a variety of three dimensional astrophysical problems, involving compressible hydrodynamics, due to its Lagrangian nature and also its remarkably-low computational cost in comparison to other techniques that involves 3D-grids or finite elements. The technique combines some properties of the distribution theory with the Monte Carlo approach of estimating multiple integrals. Essentially, SPH reduces the hydrodynamic equations of motion to an N-body problem by using particles to transport local average (smoothed) fluid properties. A more extended review on SPH is given by Monaghan (1992) and Benz (1990).

The present SPH code has the main features of the TreeSPH class (Hernquist \& Katz 1989; hereafter HK89). Initially, the code was designed to run on vector machines. Thus, the structure and methods for the octal-tree construction, and even for the tree traversals, are based on some works in literature, regarding vectorization of tree codes (Barnes 1990; Hernquist 1990; Makino 1990). The evolution of the fluid quantities are performed by means of a modified second order leapfrog, conveniently adapted to deal with multiple time scales: more energetic particles are time integrated along smaller time-steps, while less energetic particles are integrated along larger time-steps so that orbits are syncronized according to a binary hierarchy of time-steps.

The astrophysical application for the present code is performed by studying three special cases of collision of two identical molecular clouds. This application was motivated by the fact that collisions of 
interstellar clouds, or cloud fragments, occur in a variety of astrophysical situations, and there is evidence that they can trigger fragmentation and, consequently, star formation (e.g., Bash 1979; Greaves \& White 1991; Icke 1979; Koo et al. 1994). Examples range from the dynamical support of giant molecular clouds in spiral arms (e.g., Bash 1979; Elmegreen 1989, 1990, 1992), to clump-clump collisions inside a molecular cloud. The possible outcomes of cloud collisions, like coalescence, fragmentation, or star formation, depend on many parameters, e.g., relative velocity, impact parameter, details of the cooling mechanism, which in turn depends on metallicity etc. This way, modeling fragmentation by cloud collision requires more complex terms enclosing physical parameters in the fluid equations of motion.

Both thermal energy equation and molecular cooling have the same treatment proposed by Monaghan \& Lattanzio (1991, hereafter ML91). Moreover, the Dalgarno \& McCray (1972, hereafter DM72) model is adopted to cool down faster the initial phases of the supersonic shock. In addition, we take into account the heat mechanism by cosmic rays and by the production of $\mathrm{H}_{2}$ on the surface of dust grains.

The inclusion of magnetic effects in collision experiment shall be presented as the second part of this work in the next paper.

\section{Numerical techniques and gas dynamics}

The present work adopts the traditional isotropicallyadaptive SPH, where the smoothing length is proportional to the radius of a spherical region inside which there is a fixed number of nearest particles. However, more refined approaches of adaptive interpolation, regarding anisotropy of the mass distribution, may be seen in the literature (e.g., Fulbright et al. 1995; Owen \& Fisher 1994; Owen et al. 1998; Shapiro et al. 1996).

We adopted the B-spline (Monaghan \& Gingold 1983) to model the smoothing kernel, defined in 3-dimensions as:

$W_{h}(r)=\frac{3}{4 \pi h^{3}} \begin{cases}\frac{4}{3}-2 \frac{r^{2}}{h^{2}}+\frac{r^{3}}{h^{3}}, & 0 \leq r<h, \\ \frac{1}{3}\left(2-\frac{r}{h}\right)^{3}, & h \leq r<2 h, \\ 0, & r \geq 2 h .\end{cases}$

From the equation above, the smoothing length $h$ can be calculated as half the radius of a spherical region, enclosing a fixed number of the nearest neighbors of a given particle, placed in position $\boldsymbol{r}$.

We calculate the smoothed particle gradient of any fluid parameter $A$, for a particle $i$, by using the traditional form:

$\langle\nabla A\rangle_{i}=\sum_{j} \frac{m_{j}}{\rho_{j}} \nabla_{i} W_{i j} A_{j}$, where any particle density, $\rho_{i}$, is estimated by

$\rho_{i}=\sum_{j} m_{j} W_{i j}$

and the index $j$ points to the particles in a list of the nearest neighbors of particle $i$, separated by distances smaller than a given radius. For the present kernel, this radius is naturally $2 h_{i}$.

The two-particle smoothing kernel, $W_{i j}$, appearing in equations above is the symmetrized gather-scatter form, proposed by Hernquist \& Katz (1989):

$W_{i j} \equiv \frac{W\left(\left|\boldsymbol{r}_{i}-\boldsymbol{r}_{j}\right|, h_{i}\right)+W\left(\left|\boldsymbol{r}_{i}-\boldsymbol{r}_{j}\right|, h_{j}\right)}{2}$

with $W(|\boldsymbol{r}|, h)$ given by Eq. (1).

\subsection{Momentum conservation}

The usual form of writing the self-gravitating SPH momentum equation requires a symmetrized two-particle combination of pressure and artificial viscosity (see, e.g., Hernquist \& Katz 1989; Monaghan 1992; Benz 1990):

$\frac{\mathrm{d} \boldsymbol{v}_{i}}{\mathrm{~d} t}=-\sum_{j} m_{j} \nabla_{i} W_{i j}\left(\frac{p_{i}}{\rho_{i}^{2}}+\frac{p_{j}}{\rho_{j}^{2}}+\Pi_{i j}\right)-\nabla_{i} \Phi_{i}$

where $\nabla_{i} \Phi_{i}$ is the acceleration of gravity on particle $i$, and $\Pi_{i j}$ is the Monaghan-Gingold artificial viscosity (Monaghan \& Gingold 1983):

$\Pi_{i j}= \begin{cases}\frac{\alpha \bar{c}_{i j} \mu_{i j}+\beta \mu_{i j}^{2}}{\bar{\rho}_{i j}}, & \text { if } \boldsymbol{v}_{i j} \cdot \boldsymbol{r}_{i j}<0, \\ 0, & \text { otherwise, }\end{cases}$

with

$\mu_{i j}=-\frac{\boldsymbol{v}_{i j} \cdot \boldsymbol{r}_{i j}}{\bar{h}_{i j}} \frac{1}{r_{i j}^{2} / \bar{h}_{i j}^{2}+\eta^{2}}$,

and the following convention is assumed: $a_{i j} \equiv a_{i}-a_{j}$ and $\bar{a}_{i j} \equiv 0.5\left(a_{i}+a_{j}\right)$. The coefficient $\eta$ is usually 0.1 , and the coefficients $\alpha \sim 1$ and $\beta \sim 2$. However, we heuristically found the values $\alpha=3, \beta=5$, to be a good choice to stabilize the hierarchical integration scheme. For lower values, the solutions diverged on the critical phase of the shock in an adiabatic experiment of 3-D spherical collapse, shown in Appendix C.

The coefficient $\bar{c}_{i j}$ in Eq. (6) is usually defined as the arithmetic mean of the adiabatic sound speed, $c_{\mathrm{S}}$, for particles $i$ and $j$ respectively. However, we found that the artificial viscosity works efficiently if we use the exact definition of $c_{\mathrm{S}}$ in adiabatic shocks but it allows particle interpenetration if we adopt the present molecular mixture and cooling, mainly in the post-shocking layers where it has cooled down to lower temperatures as $\sim 10 \mathrm{~K}$. The main reason for this effect is that a considerable reduction in the specific thermal energy occurs at lower temperatures, which reduces considerably the local sound speed. We minimized this effect replacing $c_{\mathrm{S}}^{2}$ by the equivalent 
specific thermal energy of a (non-physical) $100 \%$ dissociated hydrogen cloud:

$u_{\mathrm{H}}=\frac{3}{2}\left(X+\frac{Y}{4}\right) R_{\mathrm{o}} T+\frac{X D_{\mathrm{o}}}{2 m_{\mathrm{H}}}$,

so that $\bar{c}_{i j}=0.5\left(u_{\mathrm{H}_{i}}+u_{\mathrm{H}_{j}}\right)$, where $D_{\mathrm{o}}, X$ and $Y$ are discussed below (Sect. 2.2).

Monaghan (1989) discussed an alternative solution to the problem of particle penetration in SPH. However, we have not adopted such device since the integration scheme has shown numerical instability on supersonic shocks in our calculations.

\subsection{Chemical equilibrium and thermal energy}

The interstellar clouds in our experiments are considered a mixture of atomic and molecular hydrogen, helium and traces of $\mathrm{CO}$. Only $\mathrm{H}, \mathrm{H}_{2}$ and $\mathrm{He}$ contribute effectively to the thermal energy; the role of $\mathrm{CO}$ is limited to the cooling of the gas, at temperatures lower than $10^{4} \mathrm{~K}$ and at high densities. We assume that the chemical abundance (in mass) is $X=0.75$ and $Y=0.25$. The chemical equilibrium between $\mathrm{H}$ and $\mathrm{H}_{2}$ is the same of ML91, which is only valid in dark regions. The relative abundance, $y$, of atomic $\mathrm{H}$ obeys the equilibrium equation:

$\frac{y}{1-y}=\frac{2.11}{\rho X y} \mathrm{e}^{-D_{\circ} / k T}$,

where

$y=\frac{\rho(\mathrm{H})}{\rho X}$,

and $D_{\mathrm{o}}=4.477 \mathrm{eV}$ is the dissociation energy of $\mathrm{H}_{2}$ molecule. The specific thermal energy for the gas mixture is given by

$u(T)=\frac{3}{2} \frac{R_{\mathrm{o}}}{\mu} T+\frac{X y D_{\mathrm{o}}+X(1-y) E\left(\mathrm{H}_{2}\right)}{2 m_{\mathrm{H}}}$,

and the equation of state is given by

$\frac{p}{\rho}=\frac{R_{\mathrm{o}}}{\mu} T$.

with $\mu$ being the mean molecular weight:

$\frac{1}{\mu}=\left(\frac{1+y}{2}\right) X+\frac{Y}{4}$.

The function $E\left(\mathrm{H}_{2}\right)$ is the mean internal (nontranslational) energy of the $\mathrm{H}_{2}$, given by

$E\left(\mathrm{H}_{2}\right)=f_{\mathrm{rot}}+\frac{k \theta_{\mathrm{vib}}}{\exp \left(\theta_{\mathrm{vib}} / T\right)-1}$,

where $f_{\text {rot }}$ is the rotational internal energy, that we extracted from Eq. (3.9) of ML91, and $\theta_{\text {vib }}$ is $6100 \mathrm{~K}$.

Temperatures are calculated by iterations from Eq. (11) predicting more accurate values by solving $T$ from the linear term. In general, convergence is reached within a good tolerance $\left(\sim 10^{-4}\right)$ after two or three iterations.

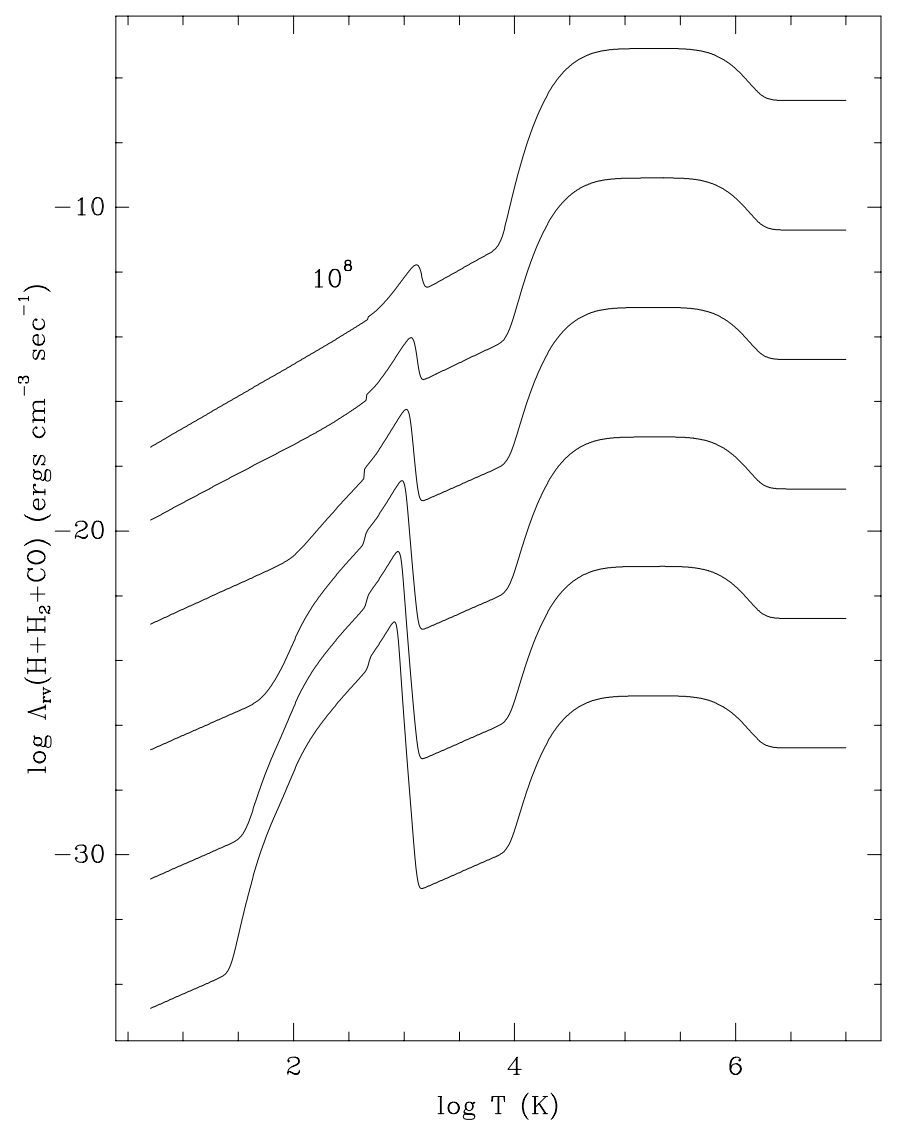

Fig. 1. The total cooling rate in ergs $\mathrm{s}^{-1} \mathrm{~cm}^{-3}$ for the mixture $\mathrm{CO}, \mathrm{H}_{2}, \mathrm{HI}$ and HII given in Eq. (17) (see text) for six different number densities of hydrogen species: $10^{-2}, 10^{0}, 10^{2}, 10^{4}, 10^{6}$, $10^{8} \mathrm{~cm}^{-3}$

\subsection{Energy conservation. Cooling and heating}

The particle form of the first law of thermodynamics is expressed as

$\frac{\mathrm{d} u_{i}}{\mathrm{~d} t}=\frac{1}{2} \sum_{j} m_{j} \boldsymbol{v}_{i j} \cdot \nabla_{i} W_{i j} \Pi_{i j}-\mathcal{L}_{i}$,

where we define $\mathcal{L}_{i}$ as the rate of specific energy loss:

$\mathcal{L}_{i}=\frac{\Lambda_{i}-\Gamma_{i}}{\rho_{i}}$.

$\Lambda_{i}$ and $\Gamma_{i}$ being the cooling and heating functions, respectively.

The cooling model takes into account two main processes that dominate in different ranges of temperature. Below $10^{4} \mathrm{~K}$ the cooling is mostly due to molecules; we adopt the model of Hollenbach \& McKee (1979, hereafter HM79). Above $10^{4} \mathrm{~K}$, free-free emission by electrons is the main cooling mechanism, which is well described by the DM72 cooling function. In the simulations presented in this work, the temperature reached values just above $10^{4} \mathrm{~K}$.

The total expression for the specific cooling rate in $\operatorname{erg~} \mathrm{cm}^{-3} \mathrm{~s}^{-1}$ is:

$\Lambda=8.510^{-5} n_{\mathrm{H}}^{2} L(\mathrm{CO})+n\left(\mathrm{H}_{2}\right)^{2} L\left(\mathrm{H}_{2}\right)$ 
$+n(\mathrm{H}) n\left(\mathrm{H}_{2}\right) L(\mathrm{H})+n(\mathrm{H})^{2} L(\mathrm{H} \mathrm{II})$,

where $n_{\mathrm{H}}$ is the number density of hydrogen species, $n(\mathrm{H})$ is the number density of atomic hydrogen, and $n\left(\mathrm{H}_{2}\right)$ is the number density of molecular hydrogen:

$n_{\mathrm{H}}=n(\mathrm{H})+n\left(\mathrm{H}_{2}\right)$.

The functions $L(\mathrm{CO}), L(\mathrm{H}), L\left(\mathrm{H}_{2}\right)$ were taken from the work of HM79, which is well summarized by ML91. The cooling function $L(\mathrm{H}$ II $)$ is the ionized hydrogen contribution that is given by the interpolation formula of DM72:

$L(\mathrm{H}$ II $)=10^{-21}\left[10^{-0.1-1.88\left(5.23-\log _{10} T\right)^{4}}\right.$

$\left.+10^{-1.7-\Theta(T)}\right]\left(\mathrm{erg} \mathrm{cm}^{3} \mathrm{~s}^{-1}\right)$

with

$\Theta(T)= \begin{cases}0.2\left(6.2-\log _{10} T\right)^{4}, & \text { if } \log _{10} T \leq 6.2, \\ 0, & \text { otherwise. }\end{cases}$

This contribution is effective only in the initial stages of the collision experiments, where the gas temperature reaches values comparable to that one of an $\mathrm{H}$ II region.

A plot of the cooling rate $\Lambda$ as a function of the temperature, for different number densities of hydrogen species, is shown in Fig. 1. The peak around $10^{3} \mathrm{~K}$ is due to the cooling by $\mathrm{H}_{2}$, expressed by the functions $L(\mathrm{H})$ and $L\left(\mathrm{H}_{2}\right)$ reported by HM79. These functions rise strongly between $10^{2}$ and $10^{3} \mathrm{~K}$, as shown in Figs. 7 and 8 of HM79. The steep decrease of the $\mathrm{H}_{2}$ contribution at about $10^{3} \mathrm{~K}$ is due to the dissociation of $\mathrm{H}_{2}$. According to Eq. (9), the relative abundance of molecular $\mathrm{H}$, is affected by a strong transition in chemical equilibrium near $10^{3} \mathrm{~K}$. This decrease does not appear in the figures of HM79 because they are interested in the cooling per $\mathrm{H}$ species and not in the total effective cooling per $\mathrm{cm}^{3}$. The $\mathrm{CO}$ contribution is evident in Fig. 1 for high number densities and $T<10^{4} \mathrm{~K}$, since its efficiency increases with the square of the density of $\mathrm{H}$ species. One can see in Fig. 1 that the cooling law is represented by a straight line, for $T<10^{4} \mathrm{~K}$ and for $n_{\mathrm{H}}$ $\sim 10^{8}$, except for a small peak due to $\mathrm{H}_{2}$ cooling. The $\mathrm{H}_{2}{ }^{-}$ cooling profile is evident at low densities, at temperatures lower than $10^{4} \mathrm{~K}$.

The heating mechanism is assumed to be a combination of the effect of cosmic rays and of $\mathrm{H}_{2}$ production by grains, according to the expressions given by Spitzer (1978). Heating by $\mathrm{H}_{2}$ formation on dust grains is effective below a critical temperature limit, of the order of $10^{2} \mathrm{~K}$, that depends on the surface texture of the grain. There are possibly many other heating processes in the interstellar medium. For instance, one possible mechanism we have not included here is the heating due to gas-grain collisions (e.g., Spitzer 1978; Tielens \& Hollenbach 1985). We believe the mechanisms that we have taken into account are the dominant ones.

The heating by cosmic rays is given by

$\Gamma_{\mathrm{CR}} \approx 3.810^{-29} n_{\mathrm{H}}\left(\mathrm{erg} \mathrm{cm}^{-3} \mathrm{~s}^{-1}\right)$, and for the heating by $\mathrm{H}_{2}$ molecules evaporated from dust grains,

$\Gamma_{\mathrm{H} d} \approx 2.210^{-28} n_{\mathrm{H}} n(\mathrm{H})\left(\mathrm{erg} \mathrm{cm}^{-3} \mathrm{~s}^{-1}\right)$.

The total heating per unit volume is given by

$\Gamma=\Gamma_{\mathrm{CR}}+\Gamma_{\mathrm{H} d}$.

\subsection{The computer code}

\subsubsection{Basic implementation}

The present code belongs to a new class of hydrodynamic codes, called TreeSPH, which was originally developed by HK89. The main characteristic of TreeSPH is the computational reuse of the tree-descent on both gravity-calculations and neighbors-searching. Essentially, we followed the directions given by classical works in the literature (e.g., Barnes 1990; Hernquist 1990; HK89; Makino 1990) to construct the tree-subroutines.

One particular application of the SPH-interpolation technique is the program we developed to visualize the simulation results in gray scale or in pseudo-color images (cf. Figs. 6 and 7). To be visualized, each particle quantity (e.g., density, temperature etc.) is smeared out in a 2-D pixel-grid, and conveniently converted to pixel quantities ( 8 or 24-bits). As the out-put file of a given simulation stores relevant SPH information (e.g., the smoothing length $h$ ), the particle smoothing-length itself is used to cover some pixels inside a radius $r_{i}=2 h_{i} / \sigma$, where $\sigma$ (in length unit per pixel) is the grid scale-factor. It may also be used to control the image resolution. The interpolation is performed with particles that obey some criterion (e.g., with densities or temperatures in a given range, $z$ coordinate tolerance to define a slide etc.).

A more complete version of the code, including a more complex cooling for the mixture $\mathrm{H}, \mathrm{H}_{2}, \mathrm{OH}, \mathrm{H}_{2} \mathrm{O}$, CO, with the $\mathrm{H}, \mathrm{H}_{2}$ and $\mathrm{H}_{2} \mathrm{O}$ chemistry shall be presented in a future paper.

\subsubsection{Gravity forces and neighbors searching}

Both gravity acceleration and potential field are calculated by means of the octal-tree scheme, called $\mathrm{BH}$ method (e.g., Barnes \& Hut 1986, 1989; Hernquist 1987, 1988, 1990; Makino 1990), with the quadrupole approximation properly derived for the softened potential (see Appendix A).

Essentially, the same procedure to perform gravity calculations is applied to perform the nearest neighbors searching. The difference in both implementations, besides the specific calculations, is the stop-condition for tree-descents. On gravity computations, the stop condition is the essential of the BH method, which is described in several papers concerning this matter (e.g., Barnes \& Hut 1986, 1989; Hernquist 1987, 1988). 
In the nearest-neighbors searching (NNS), a treedescent is performed in order to assemble for each particle a list of the nearest other particles that are separated with distances below or equal to a given radius $R$. The NNS tree-descent through a given cell stops if this one is unable to contain any neighbor inside, which may be translated to the following condition:

$\max \left\{\left|x_{i}-x_{\mathrm{c}}\right|,\left|y_{i}-y_{\mathrm{c}}\right|,\left|z_{i}-z_{\mathrm{c}}\right|\right\}>R+s$,

where $\left(x_{i}, y_{i}, z_{i}\right)$ is the Cartesian coordinates of the fixed particle, with generic index $i,\left(x_{\mathrm{c}}, y_{\mathrm{c}}, z_{\mathrm{c}}\right)$ is the Cartesian coordinates of cell's mass-center, and $s$ is the cell's size.

If the above condition is not satisfied, the focused cell may contain neighbors, and the tree-descent is resumed until the newly pointed cell encloses a single particle inside. This new candidate, with index $j$, is one of the nearest neighbors if the following condition is true:

$\left|\boldsymbol{r}_{j}-\boldsymbol{r}_{\mathrm{c}}\right| \leq R$

and then, this particle index is stored in the neighborslist for the fixed particle. After a complete search, each particle have their corresponding nearest-neighbors lists.

The NNS algorithm itself is applied to determine the smoothing lengths. The NNS routine returns, for particle with index $i$, a list of the nearest neighbors within an input radius $R_{i}$. If the number of neighbors found within $R_{i}$ exceeds an expected number $N_{\mathrm{f}}$, the list is sorted according to the neighbors distances, and then the exceeding particles are discarded from the neighbors-list (Heller 1993). Thus, the corrected radius is the maximum distance in the remaining neighbors- list. The smoothing length, in the case of the adopted kernel model is then $h_{i}=0.5 R_{i}$.

If the number of neighbors found by the NNS procedure is less than the expected $N_{\mathrm{f}}$, the new initial radius for neighbors search is twice the old value so that a new cycle of search-and-sort is required until a convergence criterion is satisfied. It has been adopted adopted a tolerance of \pm 2 neighbors in the present work.

For SPH computations, it is required a variant of the above algorithm to assemble a list of the nearest particles that give non-zero contribution to the smoothing kernel. This effective-neighboring condition may enlarge the list for some configurations, reaching increases of about $50 \% N_{\mathrm{f}}$. We call this algorithm the effective-neighborssearch (ENS), which consolidates the construction of the final neighbors list. Along with an ENS tree-descent for a given particle $i$, any resolved particle $j$ is assigned as an effective neighbor if the relation $d \leq 2 \max \left(h_{i}, h_{j}\right)$ is satisfied.

The adopted gravity softening is the classical Aarseth's approach, where each particle is is gravitationally equivalent to a Plummer's sphere with the same mass of the original particle and with a half-mass radius $\epsilon$. This approach does not reproduce Keplerian orbits even for a pair of particles separated far beyond the effective-radius $(d>2 \epsilon)$. This problem is overcome by the softening scheme of HK89, where the B-spline kernel itself is applied to perform the softening.

At the initialization of a new SPH (or puregravitational) experiment, the program calculates iteratively the maximum softening length $\epsilon_{\max }$ as a function of the gravity linear-scale:

$\epsilon_{\max }=\left(\frac{-G M_{\mathrm{tot}}}{E_{\mathrm{G}}}\right) N_{\mathrm{tot}}{ }^{-1 / 3}$,

where $N_{\text {tot }}$ is the total number of particles in the system, $M_{\text {tot }}$ is the system's total mass, $E_{\mathrm{G}}$ is the total gravitational energy, calculated by means of the gravitational part of the treecode itself. $\epsilon$ is then estimated as the mean interparticle spacing for an equivalent homogeneous selfgravitating system with the above characteristics.

The energy $E_{\mathrm{G}}$ depends on the chosen value for $\theta$ and the initial value for $\epsilon$, which can be zero or, if a pertinent system's linear scale $l$ is known, its initial value can be $\epsilon=l N^{-1 / 3}$ in order to accelerate the $\epsilon$-determination. Then, a new value for $\epsilon$ is returned from Eq. (26). This corrected value for $\epsilon$ can be used as a new initial value to repeat the above procedure, allowing then a more accurate value for $\epsilon$. In general, three iterations are sufficient to reach a good value for $\epsilon$.

In order to perform calculations with spatially varying softening length, we adopted the scatter form (HK89) since this allows quadrupole approximation without involving spatial derivatives of $\epsilon$. However, we shall impose an upper limit $\epsilon_{\max }$ to the softening length, either being the one calculated from Eq. (26), at the initialization of the experiment, or an input value, so that the definitive softening length is written as

$\epsilon=\min \left(\epsilon_{\max }, s\right) \geq \epsilon_{\min }$

with $s$ being the cell's size. The lower limit $\epsilon_{\min }$ is used to avoid clumps to collapse indefinitely in dissipative simulations, yielding numerically unsolvable scale-contrasts. Of course, the adaptive $\epsilon$ in Eq. (27) does not allow energy conservation since the corresponding Hamiltonian is no longer constant. However, this energy error is negligible in comparison to the high amount of energy loss due to the efficient cooling adopted in the present work. Particularly, it has been noticed that the propagated errors, adopting Eq. (27), are in general less significant than common conservation-errors of SPH simulations of adiabatic collapses. However, on simulations of pure gravitational collapse of low-velocity-dispersion spherical systems, the results were catastrophic at the critical passage of the collapse (splash). An alternative approach to perform tree-gravity calculations, with variable resolution, is presented by Nelson \& Papaloizou (1994), where the tolerance parameter, $\theta$, is halved if a given cell has distance smaller than $\epsilon$. 


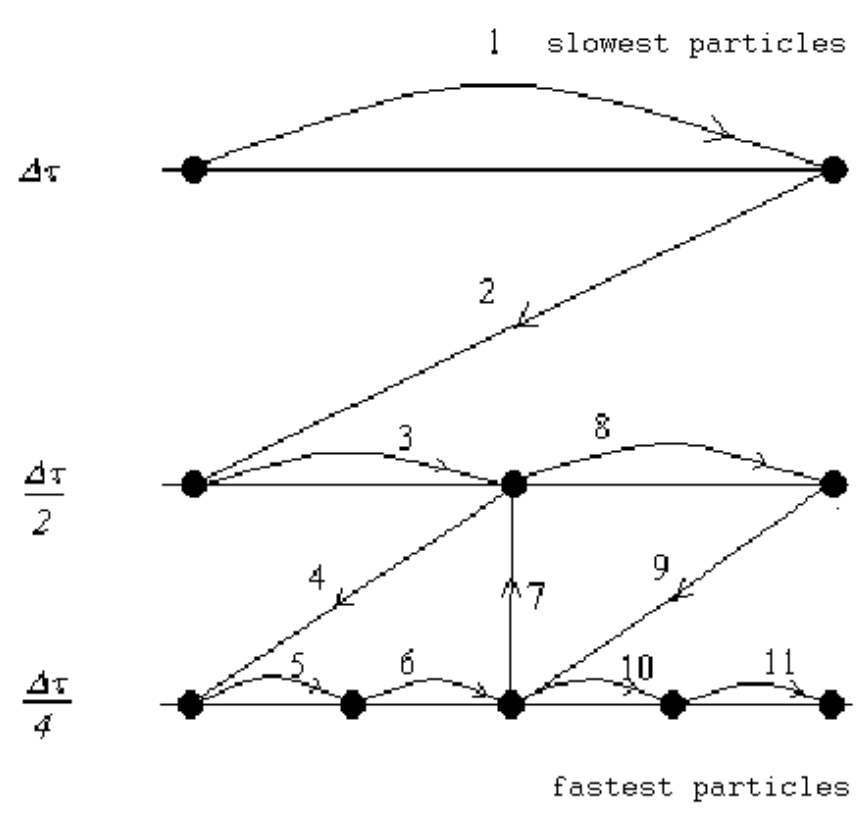

Fig. 2. The hierarchical scheme for the multiple time-step leapfrog. In the figure it is assumed the pre-fixed integration, so that upper level particles (coarser time-steps) are integrated before a descent

\subsection{The integration scheme}

\subsubsection{Time-stepping}

The multiple time-scale integration-scheme requires bookkeeping to control what particles are integrated with a given time-step, and how a time-step is selected regarding synchronization of the finite-differences solutions. One possible time scheme is the one illustrated in Fig. 2, where the finite-differences equations are solved along with a binary hierarchy of time-steps.

For each time-step, a list (time-bin) of dynamically similar particles is assigned. Each time-bin is indexed by a time-depth, $l=\{0,1, \ldots\}$, which, in turn, labels a timestep $\Delta t_{l}=2^{-l} \Delta t_{0}$, where $\Delta t_{0}$ is the root time-step that is calculated in the program initializations. For this reason, a time-step must be an ease-to-handle floating-point, so that divisions by two will not compromise the time integrity. Thus, the chosen initial time-steps must be numbers like $0.0078125,0.00390625$ etc. Numbers such as $1 / \pi$, exp -23 etc. will round-off on divisions by two, inducing synchronization breaking, and the program will hang due to infinite loops or it will at least incur in wrong results.

An upper-limit time-step is calculated from stability/accuracy considerations, which may be associated to the local physical properties. Regarding synchronization, the definitive time-step is determined as the maximum power of two that is smaller than or equal to the initial value.

For pure gravitational purposes the maximum timestep is adopted as being half of the cell's size divided by the particle's velocity (e.g., Jernigan \& Porter 1989), assuming that the octal-tree itself is the standard of rest. For SPH simulations, the maximum individual time-step is calculated by the Courant stability condition:

$\tau_{i}^{\mathrm{c}}=\frac{h_{i}}{\max \left(c_{s_{i}}, \sum_{j} \mu_{i j}, \ldots\right)}$,

so that we determine the particle time-step (time-bin) as being the maximum time-step satisfying:

$\tau_{k}=2^{-k} \tau_{\mathrm{o}} \leq \tau_{i}^{\mathrm{c}}$.

The time-stepping algorithm descends recursively to the deepest time-bin. Before a descent, a time accumulator (indexed by the current time-bin) is set equal to zero. After the descent is exhausted, the current time-step is accumulated; the particles of the current time-bin are simultaneously integrated, until the returning condition of the accumulated time for the current level equals the timestep of the parent time-bin. The time-stepping order may be changed from pre-fixed integration the post-fixed one by placing the leapfrog subroutine-call at the end of the time-bin descent subroutine-call. However, we have noticed that in some tests the post-fixed integration increases the numerical cooling in compressive shock simulations.

\subsubsection{Modified leapfrog}

The SPH equations of motion are integrated by means of a time-adaptive leapfrog, so that corrections due to the changing of time-steps are explicitly included in the finite-differences equations. A particle may jump from a time-bin to another so that along its history the particle may have visited several time-bins. The finite-differences scheme is derived in an approach similar of that given by Hernquist \& Katz (1989), so that positions and specific thermal energies are time-centered and velocities are synchronized with the time-level. Thus, if the SPH particles are dynamically described by their position vector, $\boldsymbol{x}_{n+1 / 2}$, velocity, $\boldsymbol{v}_{n}$, and specific thermal energy, $u_{n+1 / 2}$, we have the following finite difference equations:

$\boldsymbol{x}_{n+1 / 2}=\boldsymbol{x}_{n-1 / 2}+\bar{\tau}_{n} \boldsymbol{v}_{n}+\bar{\tau}_{n} \delta \tau_{n} \boldsymbol{a}_{n-1 / 2}$

$\boldsymbol{v}_{n+1}=\boldsymbol{v}_{n}+\tau_{n+1 / 2} \boldsymbol{a}_{n+1 / 2}$

and

$u_{n+1 / 2}=u_{n-1 / 2}+0.5 \bar{\tau}_{n}\left(\dot{u}_{n+1 / 2}+\dot{u}_{n-1 / 2}\right)$

where $\bar{\tau}_{n}=0.5\left(\tau_{n+1 / 2}+\tau_{n-1 / 2}\right), \delta \tau=0.25\left(\tau-\tau_{n-1 / 2}\right)$, and the time-step for the current time-bin is $\tau_{n}+1 / 2$ and the old individual time-step was $\tau_{n-1 / 2}$.

Time-centered velocities, appearing in the acceleration and in the specific thermal-energy-rate, are predicted by

$\boldsymbol{v}_{n+1 / 2}=0.5\left(\boldsymbol{v}_{n+1}+\boldsymbol{v}_{n}\right)$.

A more detailed derivation of the above leap-frog equations is given in Appendix B. 


\section{The computer experiment: Dissipative collisions of dark molecular clouds}

Each collision simulation corresponded to a different impact parameter to verify how this influence on the cloud fragmentation and on the final aspect of the collision remnants. The chosen impact parameters were, $b=0$ (headon collision), $25 \%$ off-center $(b=5 \mathrm{pc}), 50 \%$ off-center $(b=10 \mathrm{pc})$, respectively.

\subsection{Physical parameters}

The chosen physical scales for length and time were $[l]=1 \mathrm{pc}$, and $[t]=1 \mathrm{Myr}$, respectively, so that velocity unit is approximately $1 \mathrm{~km} \mathrm{~s}^{-1}$; the gravity constant was set $G=1[m]^{-1}[l]^{3}[t]^{-2}$ for which the calculated mass unit is $[m]=222.25 M_{\odot}$. Consequently, the derived physical scales for energy, power and density are $[e]=4.23010^{45} \mathrm{erg},[\dot{e}]=1.34010^{38} \mathrm{erg} \mathrm{s}^{-1}$, and $[\rho]=1.50510^{-20} \mathrm{~g} \mathrm{~cm}^{-3}=9.06510^{3} \mathrm{H} \mathrm{cm}^{-3}$, respectively.

\subsection{Initial conditions}

Each spherical cloud was arranged by Monte Carlo so that 4096 particles was distributed according to a given density law, as described in Appendix D. We adopted the $1 / r$-profile since this is an intermediate stage between homogeneous and isothermal spherical systems according to the empirical relation given by Larson (1981):

$\rho(r)=\frac{M}{2 \pi R_{\mathrm{c}}^{2}} r^{-1}$,

where the cut-off radius, $R_{\mathrm{c}}$, is $10[l]=10 \mathrm{pc}$ and the total mass $M=10[\mathrm{~m}]=2222.5 M_{\odot}$.

All particles were initially at rest relatively to the mass-center of their respective clouds. Each cloud had its mass-center placed at their respective initial positions; in Cartesian coordinates: $(-10 \mathrm{pc}, b / 2,0)$ for Cloud 1 , and $(10 \mathrm{pc},-b / 2,0)$ for Cloud 2 . The initial velocity, assigned to each particle per object was, respectively, $\left(5 \mathrm{~km} \mathrm{~s}^{-1}, 0,0\right)$ for Cloud 1 , and $\left(-5 \mathrm{~km} \mathrm{~s}^{-1}, 0,0\right)$ for Cloud 2.

Thermodynamics initial conditions were based on observed data (see, e.g., Shu et al. 1987 for a review). Both objects were initially isothermal, with temperatures of $20 \mathrm{~K}$ being a reasonable temperature for dark molecular clouds. This assumption was necessary since it is rather difficult to setup the cooling-heating balance for a nonhomogeneous self-gravitating system, by solving $\mathcal{L}_{i}=0$ for each particle in Eq. (16) and the equations of hydrostatic equilibrium. This difficulty was merely a consequence of the strongly nonlinear dependence of the cooling function on temperatures and densities. Thus, we could not avoid some transient phenomena such as the collapse

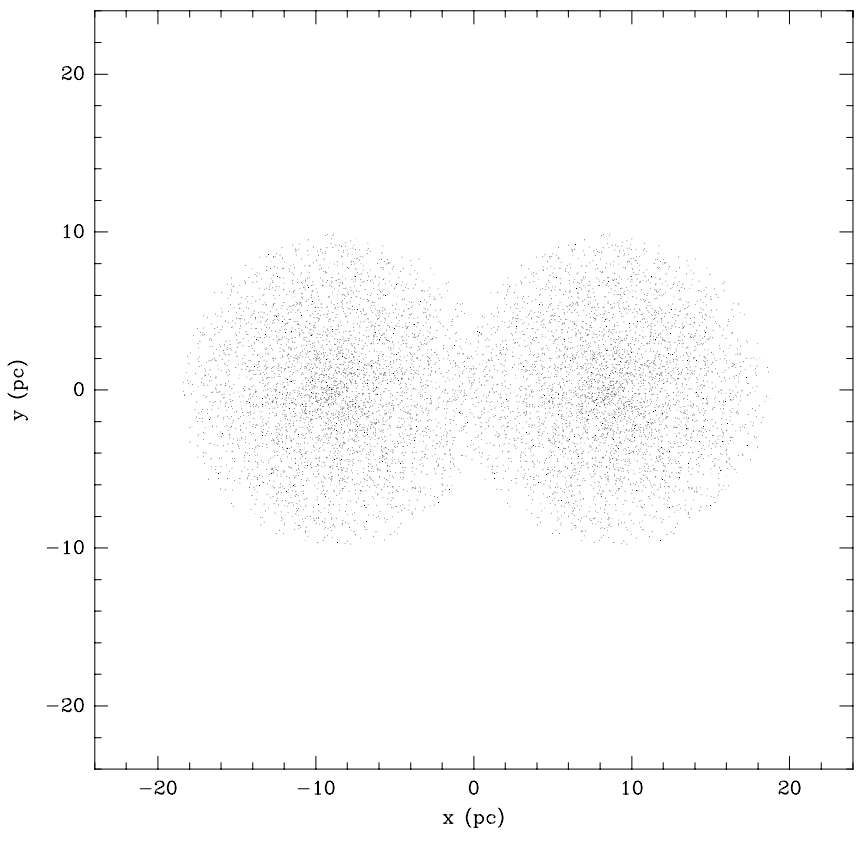

Fig. 3. Projection of the particle configuration onto the orbital plane in the head-on case after $0.25 \mathrm{Myr}$ from the initial conditions

of the inner parts due to the higher cooling-efficiency in denser regions, and a slight expansion of the outer parts due to the higher heating-efficiency by cosmic rays in comparison to the low cooling-efficiency in rarefied regions. Moreover, the clouds as a whole were initially collapsing but with a collapse time greater than their free-fall time, which is $\sim 10 \mathrm{Myr}$ in order of magnitude. However, the collision effects are in fact much more relevant than such transient events.

\subsection{SPH settings}

The fluid equations of motion were integrated with the hierarchical leapfrog, with 12 time-bins and setting the root time-step to $0.0078125 \mathrm{Myr}$. Each experiment took about $4 \mathrm{Myr}$ for head-on and $6 \mathrm{Myr}$ for off-center collisions, respectively.

The experiments ran with variable softening length. The maximum softening length, calculated from Eq. (26), was $0.5 \mathrm{pc}$, which is approximately the mean interparticle distance for $\sim 4000$ particles homogeneously distributed in a sphere of radius $10 \mathrm{pc}$. The minimum softening length was $\epsilon_{\min }=0.05 \mathrm{pc}$, which is half the mean interparticle spacing for a hypothetical situation where both clouds were crushed into a dish of about $10 \mathrm{pc}$ in radius, which is a geometrical prediction for the minimum thickness of a shock layer. This adopted value for $\epsilon_{\text {min }}$ limits Jeans instability to occur only for fragments with sizes greater than $\sim 0.05$ pc. In fact, the smallest fragments, with densities as high as $10^{4} \mathrm{~cm}^{-3}$, were $\sim 0.1 \mathrm{pc}$ in size, and they stagnated at these scales. 


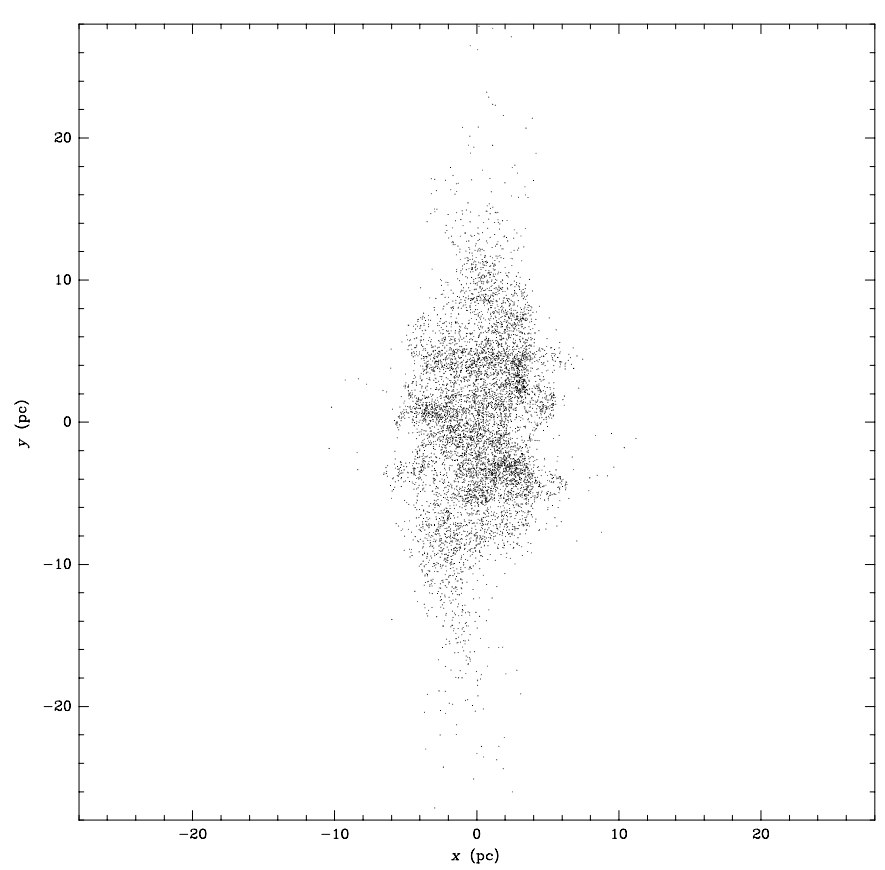

Fig. 4. Projection of the particle configuration onto the collision plane in the head-on case after 3.5 Myr. The clumped structure of the shock remnants is evident in this figure

The tolerance parameter was set $\theta=0.25$, which was a good value to guarantee both linear and angular momentum-conservation (e.g., Barnes \& Hut 1989).

The expected number of the nearest neighbors was determined heuristically regarding a better NNS performance, besides the resolution requirement. We found there is an optimal value for which the NNS is most efficient. One possible explanation for the slow convergence with a small number of neighbors may be that the statistical fluctuation in number increases as smaller is the neighborhood. For instance, if particles are locally distributed far from the Poisson's statistics, a small fluctuation in the search radius may involve a large fluctuation in the number of neighbors found. The explanation for the reduced performance with higher number of neighbors is that the NNS algorithm complexity increases approximately as $O\left(N_{\mathrm{f}} N \log N\right)$ iterations. We performed some benchmarks with different input parameters, $N_{\mathrm{f}}$, and we found an optimal choice to set $N_{\mathrm{f}}=48 \pm 2$.

From the SPH theory, the interpolation errors grow asymptotically as $O\left(h^{2}\right)$ for the present kernel (e.g., HK89), or equivalently, the interpolation is first-order accurate on $h$. From this reasoning, we may conclude that $\mathrm{SPH}$ linear contrasts are defocused by $\pm\left(N_{\mathrm{f}} / N_{\text {tot }}\right)^{1 / 3}$ from the ideal configuration. However, this factor provides a rather pessimist estimation for shock thickness since the cloud profile was far from homogeneous in all instances of the simulations. In order to globally evaluate how close an interpolation is to an ideal configuration $\left(N_{\text {tot }}=\infty\right)$, we introduced a resolution-index: $1-\left(N_{\mathrm{f}} / N_{\text {tot }}\right)^{1 / 3}$. For instance, the present choice $N_{\mathrm{f}} \approx 48$ give us a resolution

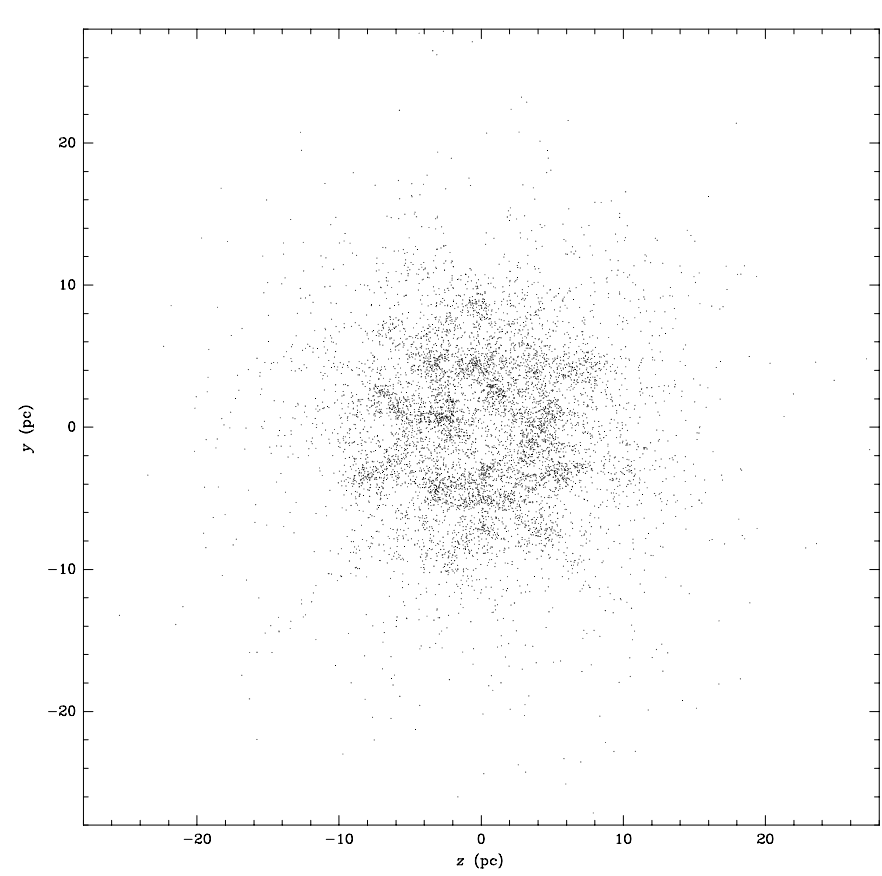

Fig. 5. The face-on projection of the same configuration illustrated in Fig. 4

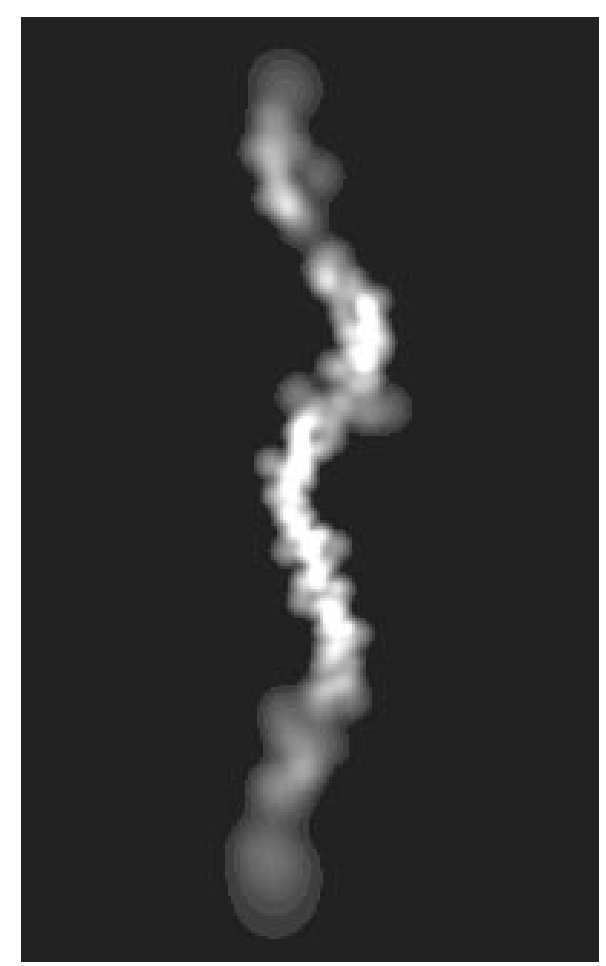

Fig. 6. Gray-scale density, averaged over a longitudinal slice (midplane) of $0.25 \mathrm{pc}$ in thickness for the head-on collision, after $\sim 1.5 \mathrm{Myr}$ from the initial conditions. Brighter tones correspond to denser regions, and darker tones correspond to rarefied regions. This figure reveals the thin aspect of the shocking region. The picture's height corresponds to $20 \mathrm{pc}$. The image is gamma corrected to saturate at the half the maximum density in the slice 


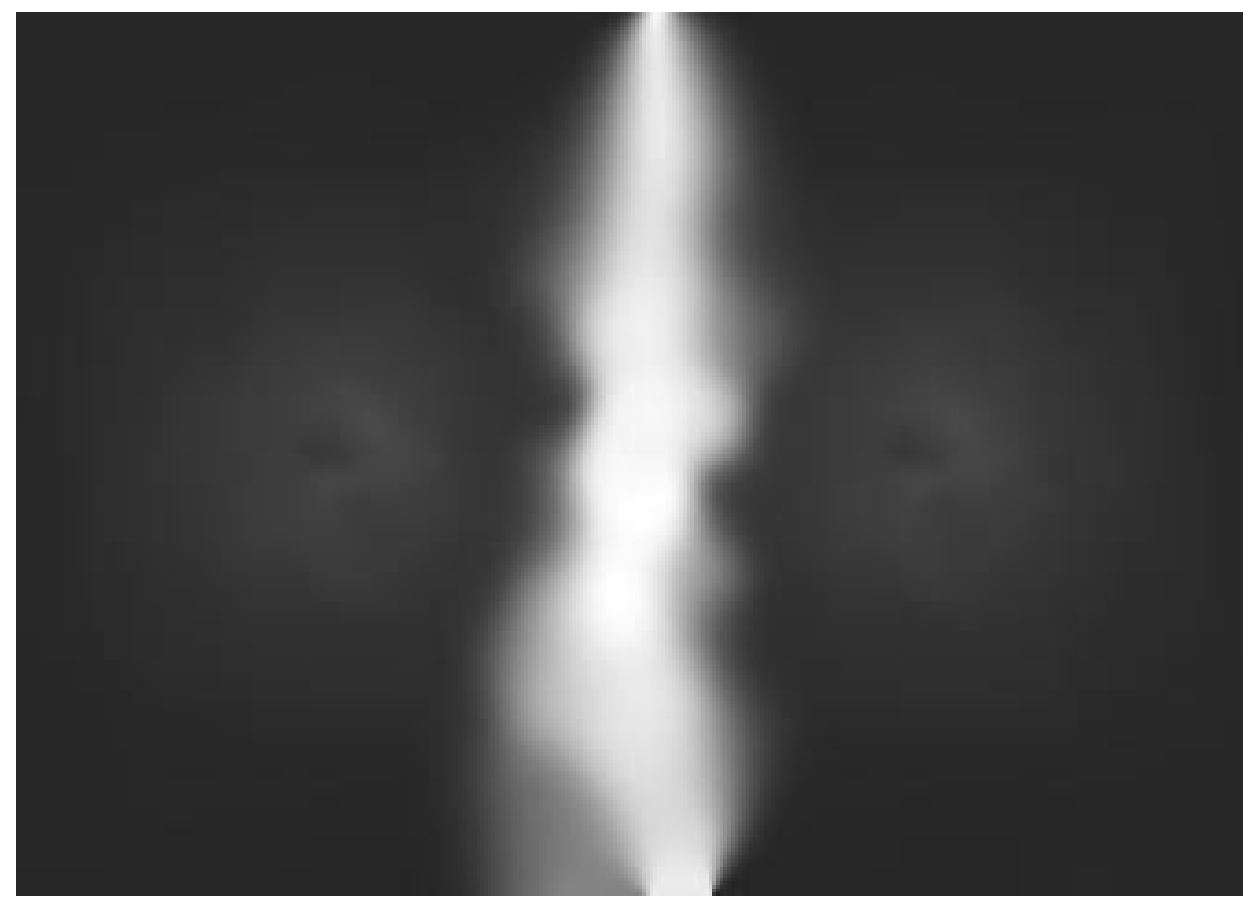

Fig. 7. Gray scale temperatures for the head-on collision $\sim 1$ Myr after the initial conditions. Brighter tones correspond to the hotter regions, and darker ones correspond to the colder regions. The darker parts at the center of both clouds have temperatures around $14 \mathrm{~K}$. The temperature of the hot spots is about $200 \mathrm{~K}$. Outer regions have temperature of $\sim 20 \mathrm{~K}$. The averaging was made within a longitudinal slice of $0.25 \mathrm{pc}$ width passing through the clouds mass center as in Fig. 6

index of $81.97 \%$, which we considered an acceptable value in comparison to several works in literature. SPH experiments with a number of particles as large as $10^{6}$ have resolution index better than $95 \%$.

The artificial viscosity input parameters were $\alpha=3$, $\beta=5$, chosen heuristically for numerical stability considerations in adiabatic tests, as discussed in Sect. 2 and we adopted the usual $\eta=0.1$.

\section{Results}

Some snapshots of the system configuration in the headon collision experiment are illustrated in Figs. 3, 4 and 5. The first frame (Fig. 3) corresponds to the system configuration after $250 \mathrm{kyr}$ from the beginning. The orbital plane for the three collisions is the $x y$-plane, with the $x$-axis containing the mass-center of both initial clouds.

In Figs. 4 and 5 respectively the face-on and edgeon configuration of the collision remnants after 3.75 Myr are shown. These plots reveal the clumped nature of the shock remnants in the head-on case. At this time, the internal parts (darker) of the remnants are approximately isothermal around $10 \mathrm{~K}$, and the outer parts remain at approximately $20 \mathrm{~K}$.

The shock density-profile at 1.5 Myr is shown in detail in the linear gray-scale as illustrated in Fig. 6. The picture was interpolated along a longitudinal slice of $0.25 \mathrm{pc}$ in thickness. The shock aspect would appear thicker if the densities were integrated along the whole line-of-sight. The density peak was $\sim 10^{3} \mathrm{~cm}^{-3}$ at the hottest configuration of the shock. Latter it reached $\sim 10^{4} \mathrm{~cm}^{-3}$ in some clumps as shown in Figs. 9 and 10.

Figure 7 reveals the temperature profile in a logarithmic gray-scale, averaged in the same longitudinal slice of Fig. 6. In Fig. 8, corresponding to the same situation of Fig. 7, we can see in more detail that each cloud has a core forming in its center. These two proto cores are approximately isothermal, with $T \approx 14 \mathrm{~K}$. The hottest spot corresponds to $\sim 200 \mathrm{~K}$. After $3 \mathrm{Myr}$ the shocked region becomes cooler than the surrounding gas. This behavior is mainly due to the strong dependence of the $\mathrm{CO}$ and the $\mathrm{H}_{2}$ cooling on density. The Dalgarno and McCray model was relevant in the first two steps of the experiments, mainly the head-on, where temperatures reached values as high as $\sim 10^{3} \mathrm{~K}$. The temperatures in the shock front reached a maximum of approximately $3000 \mathrm{~K}$. Exceptionally, a unique particle reached, along these two initial steps, a temperature of approximately $5000 \mathrm{~K}$.

We analyzed structures (e.g., clumps, shock etc.) by exhaustively creating subsets of the original output files, sorted by density, temperature, $z$-axis etc., so that we could visually identify structures that appeared in the graphs for a given range of the control parameter (e.g., density). From this preliminary analysis, we could isolate some relevant structures as those shown in Fig. 8.

After the shock extinction, the head-on collision remnants revealed a clumped aspect as shown in Figs. 4 and 5 . Some cores formed in the denser parts of the formed 


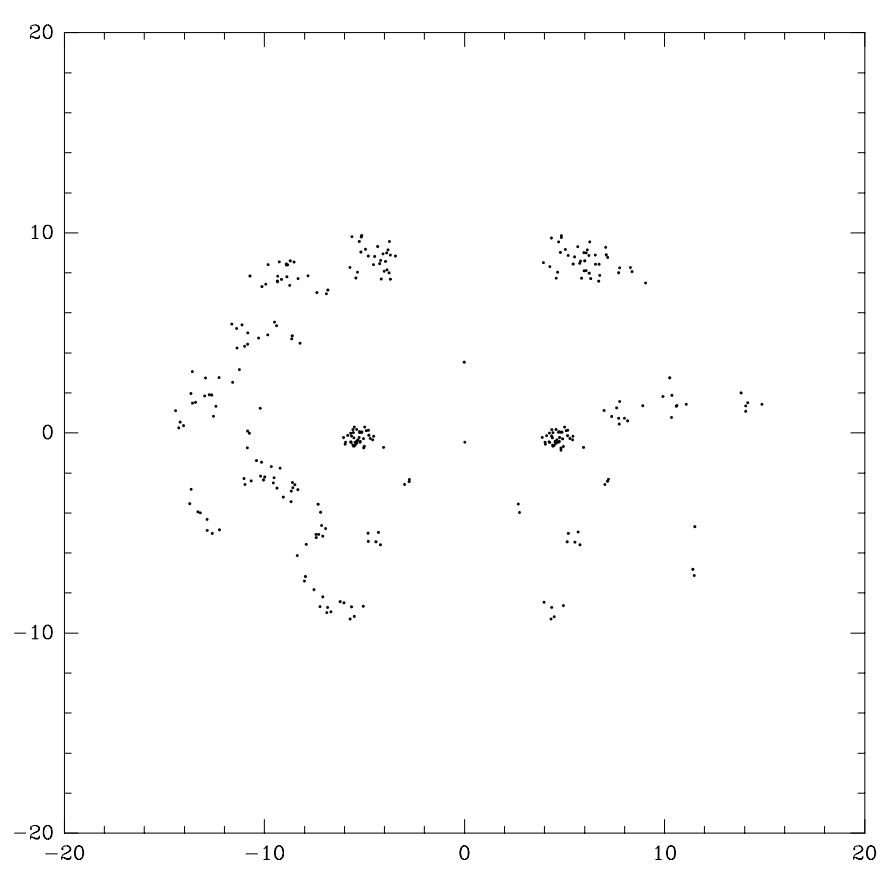

Fig. 8. Particle configuration corresponding to the situation in Fig. 7 but showing only particles with temperatures below $16 \mathrm{~K}$. This plot reveals a core forming in the central parts of each cloud

system: Fig. 9 detached the denser parts of Fig. 4 and Fig. 10 shows a zoom of the densest core of Fig. 9, where only particles with densities greater than or equal to half the maximum density $\left(1.85910^{4} \mathrm{~cm}^{-3}\right)$ are shown. All particles shown in Figs. 9 and 10 have the same temperature $T=11.68 \mathrm{~K}$, which is colder than its surroundings.

The off-center collisions were performed with two distinct impact parameters, $b_{1}=5.0 \mathrm{pc}$ ( $25 \%$ off-center) and $b_{2}=10 \mathrm{pc}$ ( $50 \%$ off-center $)$, where the maximum impact parameter for the present work is naturally $b=20 \mathrm{pc}$. The $50 \%$ off-center collision was found qualitatively similar to the previous case. However, the effects of the shock propagated by the collision on the individual cloud structure was significantly small than in the $25 \%$ off-center case, so that only the latter is illustrated here (Figs. 12 and 13).

The rate of energy loss (cloud luminosity) in the three experiments is shown as a function of time in Fig. 14. Each plot reflects the energy-loss dependence on the impact parameter. The luminosity variation is smoother for offcenter collisions. The head-on collision exhausted about $83 \%$ of the initial kinetic energy against $38 \%$ in the $50 \%$ off-center case. We observed that, although the thermal energy reached by the head-on case is greater than the one reached by the off-center collision, the head-on case became colder after about 3.5 Myr. Stronger collisions produce denser regions, which cool faster since the cooling mechanism depends strongly on density. The shock behavior was essentially driven by the cooling mechanism and by the dependence of the $\mathrm{H}_{2} \rightarrow \mathrm{H}$ transition on the

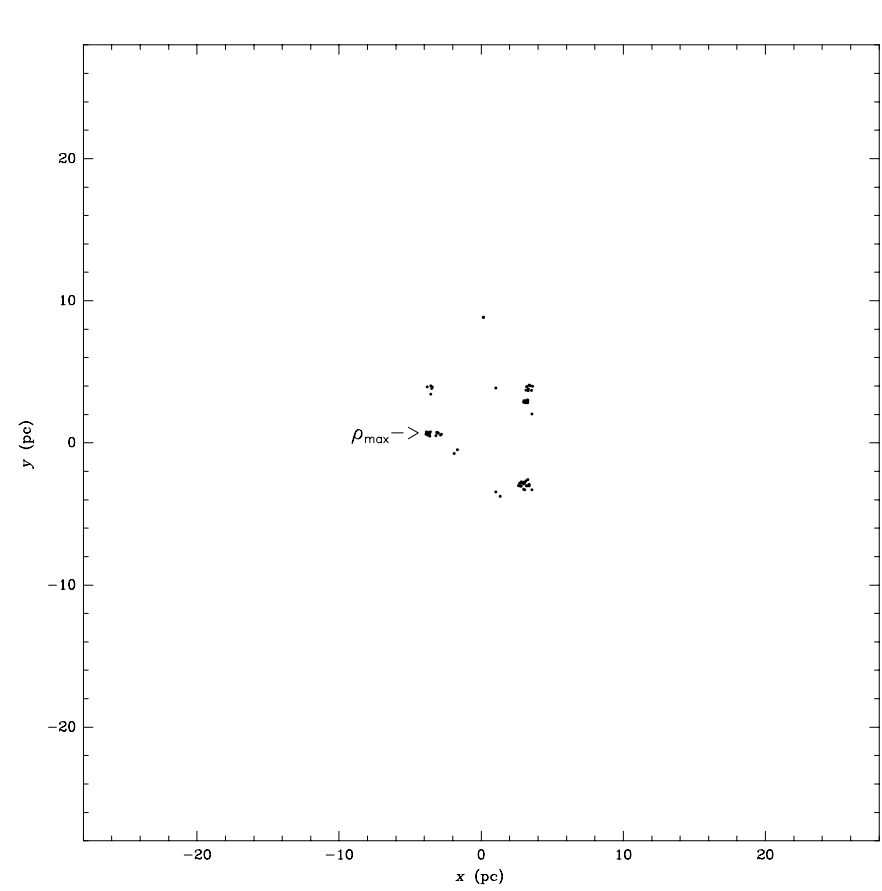

Fig. 9. Particle configuration corresponding to the situation in Fig. 4 but showing only particles with number densities greater than or equal to half the maximum density $\left(1.85910^{4} \mathrm{~cm}^{-3}\right)$. The cores correspond to the central parts of the concentrated structures in Fig. 4. All particles in this plot have the same temperature of $11.68 \mathrm{~K}$, almost half the surrounding particles

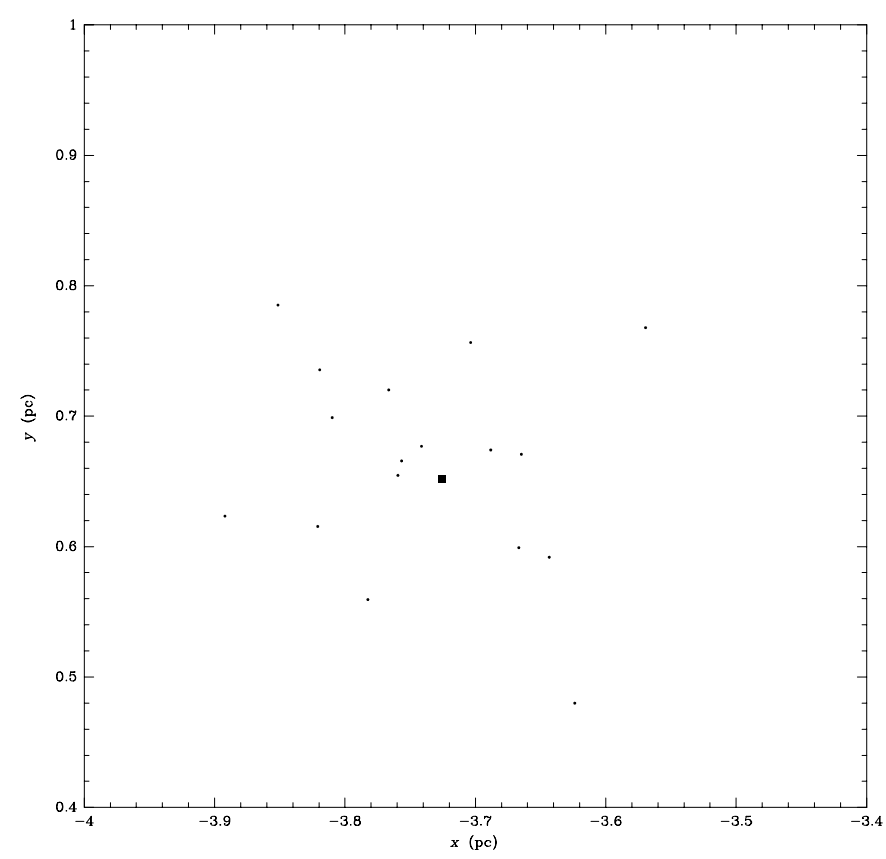

Fig. 10. A zoom in the densest core of the cluster showed in Fig. 9. The maximum number-density particle is plotted with a black square, which values $n_{\max }=1.85910^{4} \mathrm{~cm}^{-3}$. The selected particles have densities in the range $n \geq 0.5 n_{\max }$. The above structure has approximately a radius ( $\mathrm{rms}$ ) of $\sim 0.1 \mathrm{pc}$ 


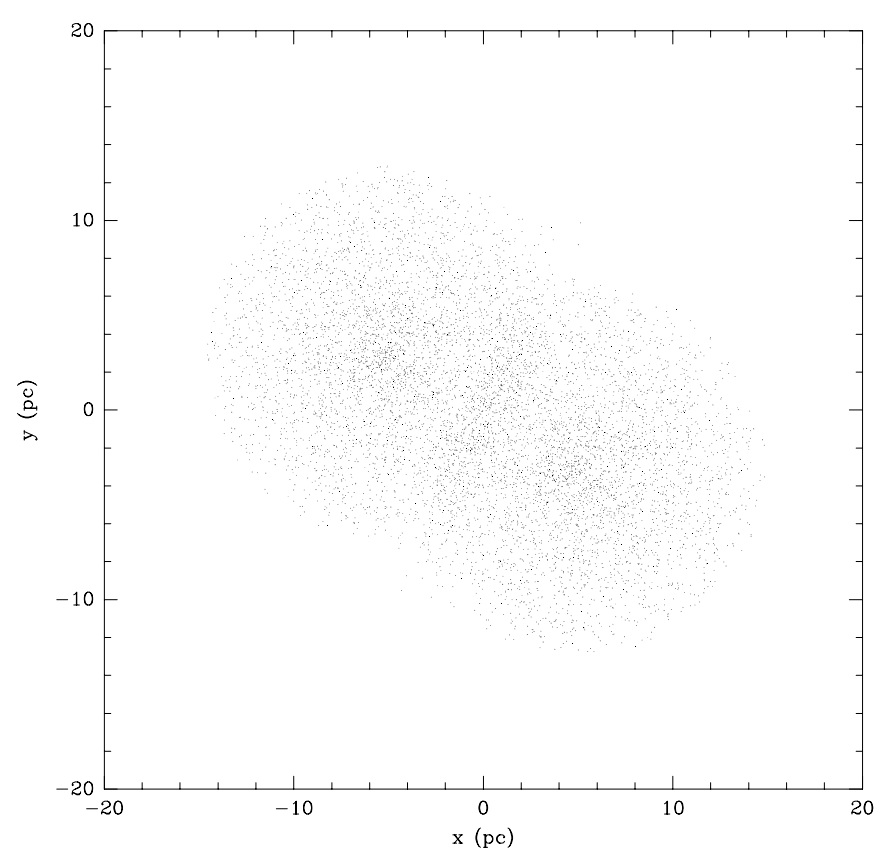

Fig. 11. 2D Plot of the particle configuration projected onto the orbital plane for the experiment with impact parameter of $5 \mathrm{pc}$ ( $25 \%$ off-center) after $0.25 \mathrm{Myr}$

temperature. The shock resolution was limited by the kernel's width, which depends on the number of neighbors.

\section{Conclusion}

The present experiment showed results with a considerable range of density-contrast. While the total size of the studied region was about $40 \mathrm{pc}$, and the average density about 100 particles $\mathrm{cm}^{-3}$ in the experiments, structures as small as $10^{-1} \mathrm{pc}$, and densities of $\sim 10^{4} \mathrm{~cm}^{-3}$ were observed.

This performance was achieved with the aid of a gather-scatter smoothing length, a scatter variable softening of the gravity field, and an adaptive integrationscheme according to the locally required time-resolution. The adoption of the scatter softening length was done in detriment of energy conservation. However, this energy error was negligible in comparison to the high amount of energy loss due to the efficient cooling.

In the first steps of the experiment, the gravity was almost irrelevant on the formation of the shock. In this phase, the Dalgarno \& McCray model was decisive on the fast cooling of the shock front and its kinetic energy dissipation. In the subsequent steps, as the shock front cooled considerably, the molecular cooling was preponderant. The strong contraction of both clouds onto almost a surface in the head-on experiment was then merely a conversion of kinetic energy into radiation due to the cooling efficiency, This efficiency increased as the density increased (shown in Fig. 1), which consequently allowed

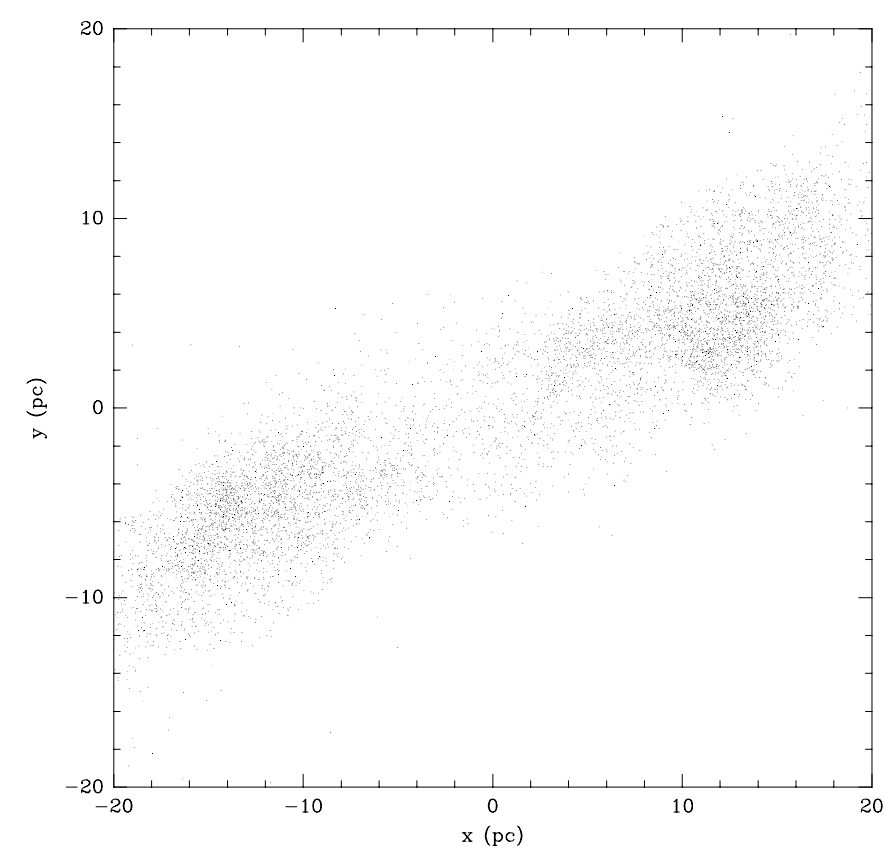

Fig. 12. 2D Plot of the particle configuration projected onto the orbital plane for the experiment with impact parameter of $5 \mathrm{pc}$ ( $25 \%$ off-center) case after $\sim 6 \mathrm{Myr}$

thermal instability along with the critical phases of the shock. Just in this phase density fluctuations were amplified, resulting in high-density peaks in the clumped configuration of Figs. 4 and 5.

The experiments demonstrate that clumps formation are strongly stimulated by supersonic cloud-cloud collisions, mainly due to the thermal instability. The main reason for the differences between our results and previous works is that the efficient cooling due to molecules at temperatures below $3000 \mathrm{~K}$ was not usually taken into account (e.g., Lattanzio et al. 1985; Lattanzio \& Henriksen 1988). This cooling mechanism for higher temperatures was relevant in the first two steps of the collision, where the hotter parts of the shock reached temperatures of the order of $1000 \mathrm{~K}$ and then dropped fastly down to temperatures comparable to $100 \mathrm{~K}$.

During the collision, the shocked layer becomes cooler than its surroundings. Consequently, the gas continuously accretes on both sides of the shocked layer. The time-scale for re-expansion of the gas after the end of the shock, in the head-on case, is found to be larger than the one obtained by Lattanzio \& Henriksen (1988) in a similar situation; the efficient energy dissipation prevents re-expansion.

It seems that the dense molecular layer, produced by the shock, survives as long as it is maintained by the extra external pressure of the accumulated gas, and starts to dissipate as soon as the shock ends. In this sense, the dense molecular phase is possibly not an equilibrium phase of the interstellar medium at normal pressures. Encounters between molecular clouds seem to be an efficient mechanism to build up enough interstellar matter to reach densities of 


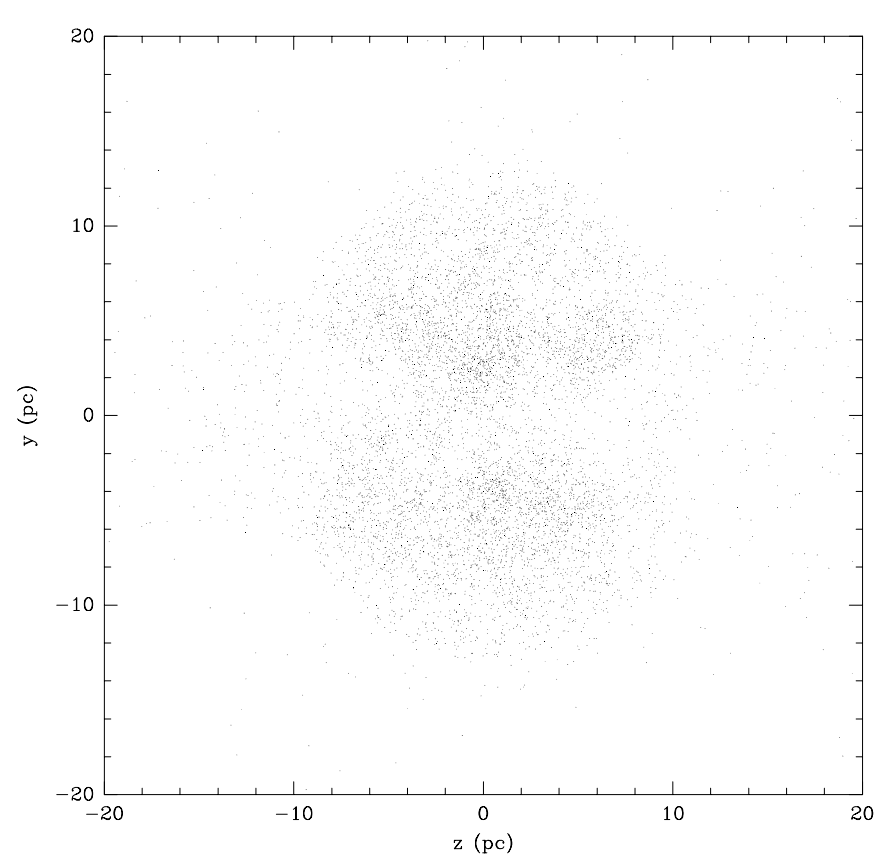

Fig. 13. The corresponding situation of Fig. 12 but projected in a perpendicular direction to the orbital plane

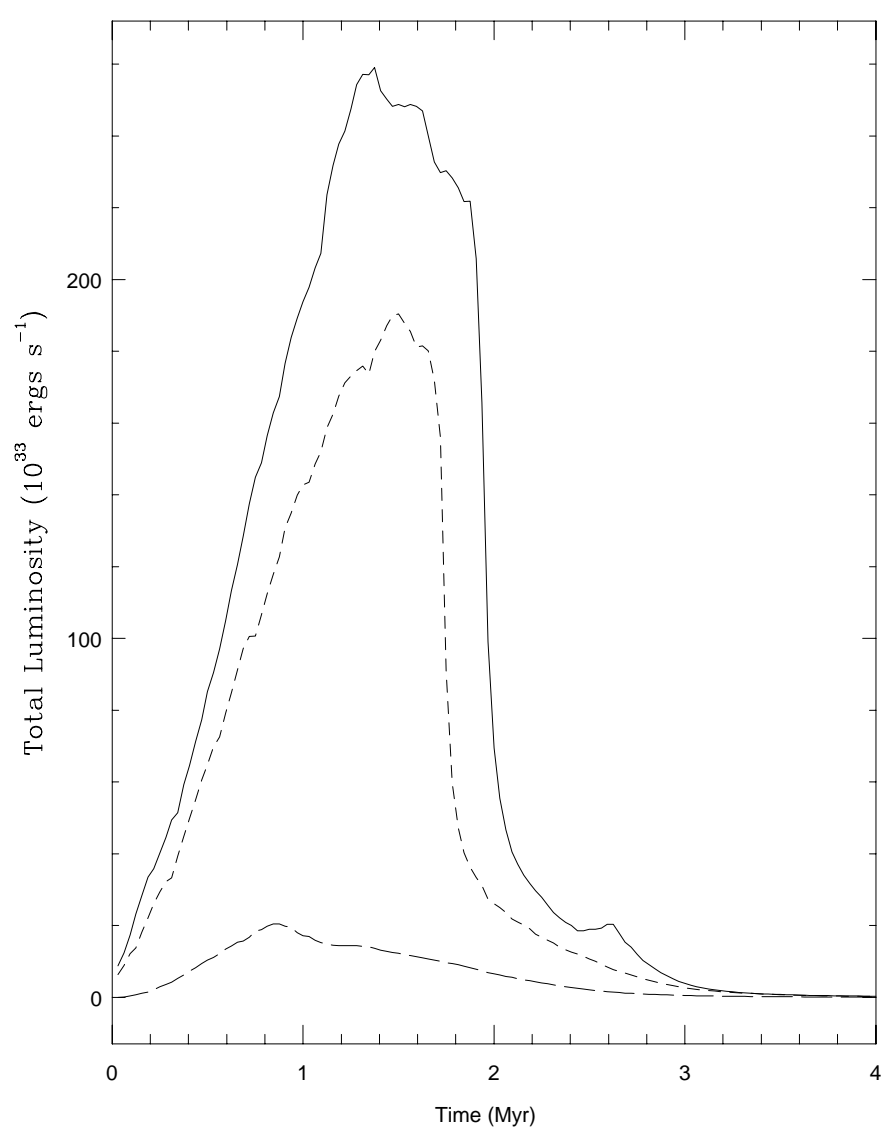

Fig. 14. The rate of energy loss (cloud luminosity) in the three experiments as a function of time. As in Fig. 9, the solid line corresponds to the head-on case, the short-dashed line to the $25 \%$ off-center case, the long-dashed line to the $50 \%$ off-center $\sim 10^{4} \mathrm{~cm}^{-3}$. If indeed molecular clouds are produced and maintained by collisions, we suggest that most of them could be geometrically thin, like the flat dense layers observed in our experiments.

Acknowledgements. E.P. Marinho is grateful to Fundação de Amparo à Pesquisa do Estado de São Paulo (FAPESP), SP Brazil, by a financial support under contract number $97 / 6157$ 4 and to Carmen Maria Andreazza by useful suggestions and a critical reading of the manuscripts. We would like to thank our referee James Michael Owen for his constructive criticisms and advices on an earlier version of this paper.

\section{Appendix A: Quadrupole expansion for the softened potential}

The correct quadrupole expansion for the softened potential may be derived by considering the potential contribution from the enclosed mass in a cubic cell of the octal-tree. By Taylor expanding the softened potential, $\tilde{\Phi}$, around the cell's mass-center, we have:

$\tilde{\Phi}=\tilde{\Phi}_{\mathrm{o}}+\tilde{\Phi}_{2}+\ldots$

where $\Phi_{\mathrm{o}}$ and $\Phi_{2}$ are respectively the monopole and the quadrupole contributions. Additional terms are suppressed since only quadrupole approximation is required. The dipole term $\Phi_{1}$ is zero since the expansion is being taken around the cell's mass-center.

The product-of-inertia tensor, with respect to the cell's mass-center, $O$, is defined as

$\boldsymbol{J}_{O}=\sum_{j} m_{j} \boldsymbol{r}_{O j} \boldsymbol{r}_{O j}$.

where $\boldsymbol{r}_{O j}$ is the relative position of a particle $j$ with respect to the cell's mass-center.

Let $\boldsymbol{R}$ be the position vector of the cell's mass-center. For notation saving, we introduce the softened mass-center distance:

$R_{\epsilon}=\left(R^{2}+\epsilon^{2}\right)^{1 / 2}$,

where $R=|\boldsymbol{R}|$.

The softened monopole-contribution to the cell's potential is written as

$\tilde{\Phi}_{\mathrm{o}}=\frac{-G M_{\mathrm{o}}}{R_{\epsilon}}$

where, $M_{\mathrm{O}}$ is the cell mass:

$M_{\mathrm{o}}=\sum_{j} m_{j}$

By analogy to the definition of unity vector, we conveniently define the following vector:

$\hat{\mathbf{R}}_{\epsilon}=\frac{\boldsymbol{R}}{R_{\epsilon}}$

observing that $\hat{\mathbf{R}}_{\epsilon} \cdot \hat{\mathbf{R}}_{\epsilon} \leq 1$. 
For convenience, we introduce the softened quadrupole mass,

$M_{2}=\left(2 R_{\epsilon}^{2}\right)^{-1}\left(3 \hat{\mathbf{R}}_{\epsilon} \cdot \boldsymbol{J}_{O} \cdot \hat{\mathbf{R}}_{\epsilon}-\operatorname{tr} \boldsymbol{J}_{O}\right)$,

to give the quadrupole contribution to the potential in the form:

$\tilde{\Phi}_{2}=\frac{-G M_{2}}{R_{\epsilon}}$.

In the non-softened case, $\epsilon \rightarrow 0$, the quadrupole mass becomes the conventional form

$M_{2}=\frac{1}{2} \frac{\hat{\mathbf{R}}}{R} \cdot\left[3 \boldsymbol{J}_{O}-\mathbf{1}\left(\operatorname{tr} \boldsymbol{J}_{O}\right)\right] \cdot \frac{\hat{\mathbf{R}}}{R}$,

where $\mathbf{1}$ is the unity tensor, and the expression inside brackets is the non-softened case for the cell's quadrupole tensor.

The quadrupole correction is performed by applying Eqs. (A2), (A7), (A8), and extracting the gradient, to obtain the quadrupole contribution to gravity-acceleration. The six Cartesian components of the tensor $\boldsymbol{J}_{O}$ are stored in the tree data-structure along with a tree-descent, visiting all the cubic cells.

\section{Appendix B: Modified leapfrog}

Consider first the synchronized solutions, $\boldsymbol{x}_{n}, \boldsymbol{v}_{n}$, for positions and velocities, respectively, at the cycle $n$ of time integration (instant $t_{n}$ ). Suppose that the old leapfrogpositions $\boldsymbol{x}_{n-1 / 2}$, reached from the instant $t_{n}$ to instant $t_{n-1 / 2}$, is receding by a half time-step $0.5 \tau_{n-1 / 2}$, where $\tau_{n-1 / 2}$ is a given time-step to advance positions from timelevel $n-1 / 2$ to $n+1 / 2$. This time-step was estimated at time-level $n-1 / 2$, obeying some stability condition (e.g. Hilbert-Courant instability conditions).

From the above considerations, we have the following second-order approximation for positions at time-level $n$ from time-level $n-1 / 2$ :

$\boldsymbol{x}_{n} \approx \boldsymbol{x}_{n-1 / 2}+\frac{1}{2} \tau_{n-1 / 2} \boldsymbol{v}_{n}-\frac{1}{8} \tau_{n-1 / 2}^{2} \boldsymbol{a}_{n-1 / 2}$,

where $\boldsymbol{a}_{n-1 / 2}$ is the particle acceleration which depends in general on positions $\left(\boldsymbol{x}_{n-1 / 2}\right)$ and position-synchronized velocities $\left(\boldsymbol{v}_{n-1 / 2}\right)$.

Second, consider the final leapfrog-positions, $\boldsymbol{x}_{n+1 / 2}$, obtained from the initial solution $\boldsymbol{x}_{n}$ advancing from timelevel $n$ to $n+1 / 2$ by a half time-step, $\tau_{n+1 / 2}$, yielded at time-level $n+1 / 2$ :

$\boldsymbol{x}_{n+1 / 2} \approx \boldsymbol{x}_{n}+\frac{1}{2} \tau_{n+\frac{1}{2}} \boldsymbol{v}_{n}+\frac{1}{8} \tau_{n+1 / 2}^{2} \boldsymbol{a}_{n-1 / 2}$.

Adding the respective members of Eqs. (B1) and (B2), and then simplifying, we have the modified second-order leapfrog scheme for positions:

$\boldsymbol{x}_{n+1 / 2} \approx \boldsymbol{x}_{n-1 / 2}+\bar{\tau} \boldsymbol{v}_{n}+\bar{\tau} \delta \tau \boldsymbol{a}_{n-1 / 2}$, where

$\bar{\tau} \equiv 0.5\left(\tau_{n+1 / 2}+\tau_{n-1 / 2}\right)$,

and

$\delta \tau \equiv 0.25\left(\tau_{n+1 / 2}-\tau_{n-1 / 2}\right)$.

Velocities are integrated from time-level $n$ to $n+1$, which is divided into the two steps: integrating with the same half time-step, $0.5 \tau_{n+1 / 2}$, from $n$ to $n+1 / 2$, and from $n+1 / 2$ to $n+1$. After some algebra, this procedure results in the following second-order leapfrog-scheme for velocities:

$\boldsymbol{v}_{n+1}=\boldsymbol{v}_{n}+\tau_{n+1 / 2} \boldsymbol{a}_{n+1 / 2}$.

Time centered velocities may be estimated with the following first-order approximation:

$\boldsymbol{v}_{n+1 / 2}=0.5\left(\boldsymbol{v}_{n+1}+\boldsymbol{v}_{n}\right)$,

which propagates third-order error to the overall leapfrog fluid equations.

Thermal energies, or any other physical quantity obeying a first order differential equation of motion (e.g., magnetic field), may be advanced with an implicit second order scheme. Since specific thermal energy rate, $\dot{u}_{i}$, depends implicitly on particle's position and velocity, $\left(\boldsymbol{x}_{i}\right.$, $\boldsymbol{v}_{i}$ ), it is quite convenient to synchronize thermal energies with positions. Velocity terms in quantities like the velocity-divergence, artificial viscosity etc. shall be timecentered via Eq. (B7).

Considering the initial solutions for the specific thermal energy and specific thermal energy rate to be $u_{n-1 / 2}$ and $\dot{u}_{n-1 / 2}$ respectively. Time-centered solutions may be obtained by integrating from time-level $n-1 / 2$ to $n$, and receding the final solutions from time-level $n+1 / 2$ to $n$. Both solutions can be written in a second order approximation:

$u_{n} \approx u_{n-1 / 2}+\frac{1}{2} \tau_{n-1 / 2} \dot{u}_{n-1 / 2}+\frac{1}{8} \tau_{n-1 / 2}^{2} \ddot{u}_{n}$,

and

$u_{n} \approx u_{n+1 / 2}-\frac{1}{2} \tau_{n+1 / 2} \dot{u}_{n+1 / 2}+\frac{1}{8} \tau_{n+1 / 2}^{2} \ddot{u}_{n}$.

Subtracting the respective members of Eq. (B8) from Eq. (B9), we find

$$
\begin{aligned}
& u_{n+1 / 2}=u_{n-1 / 2}+\frac{1}{2}\left\{\tau_{n+1 / 2} \dot{u}_{n+1 / 2}+\tau_{n-1 / 2} \dot{u}_{n-1 / 2}\right. \\
& \left.-\frac{1}{4}\left(\tau_{n+1 / 2}^{2}-\tau_{n-1 / 2}^{2}\right) \ddot{u}_{n}\right\} .
\end{aligned}
$$

Analogously, we find $\dot{u}_{n} \approx \dot{u}_{n-1 / 2}+0.5 \tau_{n-1 / 2} \ddot{u}_{n}$ and $\dot{u}_{n} \approx$ $\dot{u}_{n+1 / 2}+\frac{1}{2} \tau_{n+1 / 2} \ddot{u}_{n}$, from which we may extract the second derivative:

$\ddot{u}_{n}=\frac{\dot{u}_{n+1 / 2}-\dot{u}_{n-1 / 2}}{0.5\left(\tau_{n+1 / 2}+\tau_{n-1 / 2}\right)}$.

Substituting $\ddot{u}_{n}$ from Eq. (B11) in Eq. (B10), and after some simplifications, we have the following differencescheme:

$u_{n+1 / 2}=u_{n-1 / 2}+0.25\left(\tau_{n+1 / 2}+\tau_{n-1 / 2}\right)\left(\dot{u}_{n+1 / 2}+\dot{u}_{n-1 / 2}\right)$. 


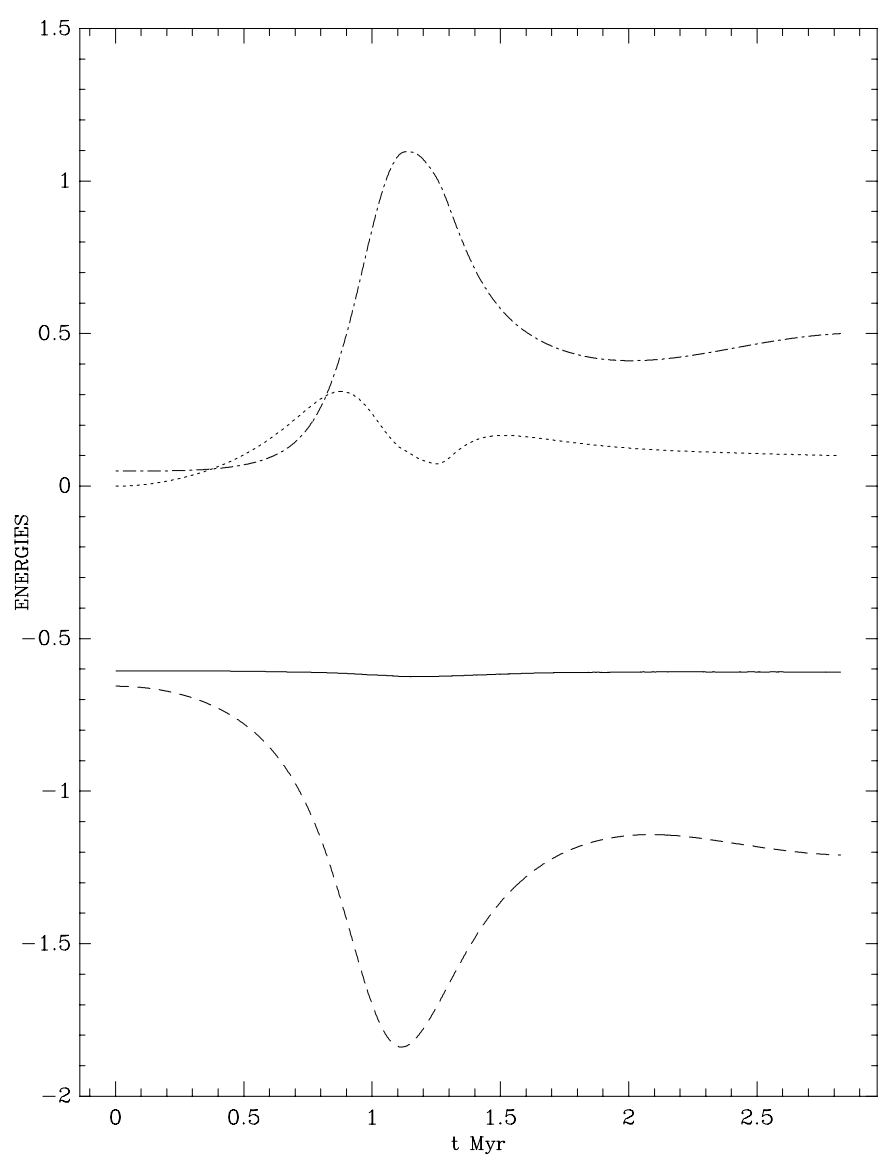

Fig. C1. The evolution of total (solid line), kinetic (doted), thermal (dash-doted) and gravitational (dashed) energies for an adiabatic collapse of 4096 particles distributed by Monte Carlo according to a $1 / r$-density profile

\section{Appendix C: Adiabatic check: Evrard's test}

One possible three-dimensional SPH energy-conservation test is the spherical collapse of an initially-cold adiabatic-gas, with a radial profile $\rho \propto 1 / r$ (Evrard 1988; HK89; $\quad$ Nelson \& Papaloizou 1994; Steinmetz M. \& Müller 1993), called Evrard's test.

The initial configuration was obtained by Monte Carlo by distributing 4096 particles spherically, according to a $1 / r$ radial-profile. As in HK89, the total mass, $M$, the cutoff-radius, $R$, and the gravitational constant $G$ are equal to unity, and the initial specific thermal-energy, $u=0.05$, was homogeneously distributed in the system. The adopted number of time-bins was 4 , starting from a root time-step, $\Delta t=0.001953125$. The softening length, $\epsilon=0.0928$, was estimated from Eq. (26), where both $E_{\mathrm{G}}$ and $\epsilon \simeq 0.1$ are iteratively calculated during the initializations. The aperture parameter $\theta$ was set as the two-bit floating $\theta=0.25$.

Thermal energy was explicitly integrated keeping fixed the entropy:

$T \frac{\mathrm{d} s}{\mathrm{~d} t}=\frac{\mathrm{d} u}{\mathrm{~d} t}+\frac{P}{\rho} \nabla \cdot \boldsymbol{v}=0$

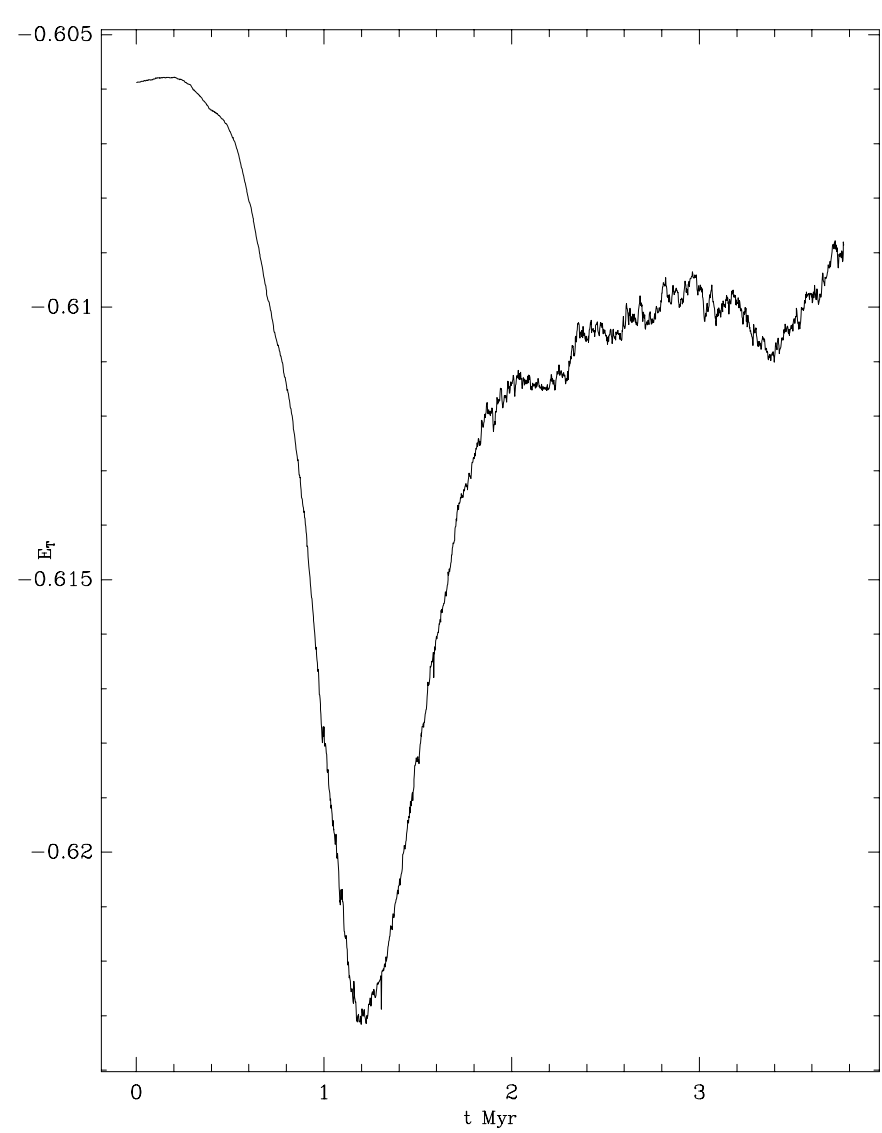

Fig. C2. A zoom in the total energy displayed in Fig. C1 revealing the conservation error in the critical phase of the adiabatic shock

so that both spatial and time inaccuracies promote an artificial heating (cooling).

Some of the results are shown in Fig. C1 in terms of the evolution of the integral energies: total, $E$, thermal, $U$, kinetic, $K$, and potential, $E_{\mathrm{G}}$. The graph in Fig. $\mathrm{C} 1$ does agree to other works in literature (e.g., HK89; SM93). The total energy was conserved within $\sim 2 \%$. The behavior of the total-energy error is illustrated in Fig. C2, showing that a numerical cooling roughly mimics the potential energy rate. Both total linear and angular momenta were better conserved than one part in $10^{5}$.

\section{Appendix D: Monte Carlo generation of spherical distributions}

Each spherical cloud was obtained from the traditional Monte Carlo scheme: three normalized random sequences $\left(\xi_{1}, \xi_{2}, \xi_{3}\right)$ are transformed to spherical coordinates $(r, \theta, \phi)$ according to the known one-particle distribution function, or to the density law $\rho(r)$. Regarding the spherical symmetry, the angular coordinates do not 
dependent on the radial coordinate. Thus, we had the radial position calculated from:

$\frac{4 \pi}{M} \int_{0}^{r} \rho(r) r^{2} \mathrm{~d} r=\xi_{1}$,

which must be solved for $r$.

The angular coordinates, $\theta$ and $\phi$, are respectively given by

$\frac{1}{2} \int_{0}^{\theta} \sin \theta \mathrm{d} \theta=\frac{1-\cos \theta}{2}=\xi_{2}$

and

$\frac{1}{2 \pi} \int_{0}^{\phi} \mathrm{d} \phi=\frac{\phi}{2 \pi}=\xi_{3}$.

\section{References}

Barnes J.E., Hut P., 1986, Nat 324, 446

Barnes J.E., Hut P., 1989, ApJS 70, 389

Barnes J.E., 1990, J. Comput. Phys. 87, 161

Bash F.N., 1979, ApJ 233, 524

Benz W., 1990, in: The Numerical Modeling of Nonlinear Stellar Pulsations Problems and Prospects, Robert Buchler J. (ed.). Publisher, Kluwer Academic Publishers, Boston, p. 269

Dalgarno A., McCray R.A., 1972, ARA\&A 10, 375

Elmegreen B.G., 1989, ApJ 347, 859

Elmegreen B.G., 1990, ApJ 357, 125

Elmegreen B.G., 1992, in: Tenorio-Tagle G., Prieto M., Sanchez F. (eds.), Star Formation in Stellar Systems III, Canary Isl\&s Winter School of Astrophysics, p. 381
Evrard A.E., 1988, MNRAS 235, 911

Fulbright M.S., Benz W., Davies M.B., 1995, ApJ 440, 254

Gingold R.A., Monaghan J.J., 1977, MNRAS 181, 375

Greaves J.S., White G.J., 1991, A\&A 248, L27

Heller C.H., 1993, ApJ 408, 337

Hernquist L., Katz N., 1989, ApJS 70, 419

Hernquist L., 1987, ApJS 64, 715

Hernquist L., 1988, Comp. Phys. Comm. 48, 107

Hernquist L., 1990, J. Comput. Phys. 87, 137

Hollenbach D., McKee C.F., 1979, ApJS 41, 555

Icke V., 1979, A\&A 78, 352

Koo B.-C., et al., 1994, ApJ 429, 233

Larson R.B., 1981, MNRAS 194, 809

Lattanzio J.C., Henriksen R.N., 1988, MNRAS 232, 565

Lattanzio J.C., et al., 1985, MNRAS 215, 125

Lucy L., 1977, AJ 82, 1013

Makino J., 1990, J. Comput. Phys. 87, 148

Monaghan J.J., Gingold R.A., 1983, J. Comput. Phys. 52, 374

Monaghan J.J., 1989, J. Comput. Phys. 82, 1

Monaghan J.J., 1992, ARA\&A 30, 543

Monaghan J.J., Lattanzio J.C., 1991, ApJ 375, 177

Nelson R.P., Papaloizou J.C.B., 1994, MNRAS 270, 1

Owen J.M., Fisher M.L., 1994, Am. Astron. Soc. Meet. 185, \#55.04

Owen J.M., Villumsen J.V., Shapiro P.R., Martel H., 1998, ApJS 116, 155

Shapiro P.R., Martel H., Villumsen J.V., Owen J.M., 1996, ApJS 103, 269

Shu F.H., Adams F.C., Lizano S., 1987, ARA\&A 25, 23

Spitzer L., 1978, Physical Processes in the Interstellar Medium. John Wiley \& Sons, NY

Steinmetz M., Müller E., 1993, A\&A 268, 391

Tielens A.G.M., Hollenbach D., 1985, ApJ 291, 722 